REVISTA INTERNACIONAL DE CIENCIAS DEL DEPORTE International Journal of Sport Science

Rev. int. cienc. deporte

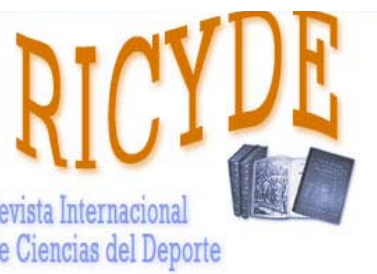

International Journal of Sport Science VOLUMEN IX - AÑO IX

Páginas:154-180 ISSN:1885-3137 No 32 - Abril - 2013

\title{
Análisis cualitativo de las implicaciones musculares de la escalada deportiva de alto nivel en competición \\ Qualitative Study of high level sport climbing muscular involvement in competition
}

\author{
Ana María de Benito, Silvia Sedano, \\ Juan Carlos Redondo y Gonzalo Cuadrado \\ Universidad de León

\section{Resumen}

El objetivo de esta investigación fue analizar las acciones musculares ejecutadas por los escaladores de alto nivel en competición. La muestra del estudio está compuesta por 72 varones (nivel técnico medio "a vista" 7C+/8A) que participaron en las pruebas de Copa de España de Escalada de Dificultad de 2009 y 2010 . Se registraron un total de 7.738 movimientos para su análisis. Se ha utilizado un método de observación validado previamente, siendo las variables de estudio: acción, posición corporal, extremidad ejecutante, tiempo empleado, zona temporal, inicio de movimiento, fin de movimiento y movimiento. Los resultados concluyen que los grupos musculares más importantes para los escaladores de alto nivel son, además de los músculos flexores del tronco, los extensores, aductores y extensores horizontales del hombro, los flexores y pronadores del codo, los extensores, aductores, abductores, rotadores internos y rotadores externos de la cadera y los extensores de rodilla. Ahora bien, el tipo de contracción muscular realizada por cada uno de ellos, dependerá del movimiento requerido. La información aquí presentada puede resultar de gran utilidad a la hora de planificar programas de entrenamiento más personalizados que mejoren el rendimiento del escalador.

Palabras clave: escalada deportiva; metodología observacional; técnica; acción muscular.

\begin{abstract}
The purpose of the current study was to analyse the muscular contractions carried out by the high level climbers in competition. The sample studied was made up of 72 male subjects ("on sight"-level 5.12c/5.13a) who participated in the Spanish Climbing Cup of 2009 and 2010. 7738 movements were registered to analyse them. The observational model used was designed and validated through a previous study. Answer levels were: action, corporal position, limb, time, time zone, start of movement, end of movement and movement. The results obtained establish that the most important muscles for high level climbers are, apart from abdominal muscles, extensors, adductors and horizontal extensors' muscles of the shoulder, flexors and rotators' muscles of the elbow, extensors, adductors, abductors, internal and external rotators' muscles of the hip and extensors' muscles of the knee. However, the type of the muscular contraction generated depends on the type of the movement required. The information given might be useful to plan more personalized training programs in order to improve the climber's performance.
\end{abstract}

Key words: sport climbing; observational methodology; technique; muscular contraction.

Correspondencia/correspondence: Ana María de Benito Trigueros

Departamento de Educación Física y Deportiva. Universidad de León. España

E-mail: ambent@unileon.es 
De Benito, A.M.; Sedano, S.; Redondo, J.C.; Cuadrado, G. (2013). Análisis cualitativo de las implicaciones musculares de la escalada deportiva de alto nivel en competición. RICYDE. Rev. int. cienc. deporte. 32(9), 154180. http://dx.doi.org/10.5232/ricyde2013.03205

\section{Introducción}

$\mathrm{L}$ a escalada, según Albesa y Lloveras (1999), se desarrolla en base a tres tipos de acciones: contracciones dinámicas concéntricas, en la mayoría de acciones en las que el cuerpo del deportista se aleja del suelo, (como por ejemplo, en una tracción para alcanzar la siguiente presa); contracciones dinámicas excéntricas, (que se producen casi siempre en situaciones de destrepe o en recobro de posiciones de reposo) y contracciones isométricas o bloqueos (que mantienen alguna parte del cuerpo fijada sin movimiento, como es el caso del mantenimiento de la mano en una presa).

Existen numerosos estudios que evalúan la fuerza muscular de los escaladores, considerando además la fuerza máxima relativa y/o la fuerza-resistencia como sus manifestaciones más determinantes en cuanto al rendimiento (Cuadrado, De Benito, Flor, Izquierdo, Sedano y Redondo, 2007; España-Romero, García-Artero, Santaliestra-Pasias, Gutiérrez, Castillo y Ruiz, 2008; España-Romero, Ortega, García-Artero, Jiménez-Pavón, Gutiérrez, Castillo y Ruiz, 2009; Grant, Hasler, Davies, Aitchison, Wilson y Whittaker, 2001; Mermier, Janot, Parker y Swan., 2000; Núñez, Edir, Viana, Gómez, Poblador, y Lancho, 2005; Poblador, Gómez y Ruíz-Alejos, 2004; Sheel, Seddon, Knight, McKenzie y Warburton, 2003; Úbeda, 2004; Watts, Daggett, Gallagher y Wilkins, 2000; Watts, Jensen, Agena, Majchrzak, Schellinger y Wubbels, 2008; Watts, Jensen, Gannon, Kobeinia, Maynard y Sansom, 2008).

Sin embargo, no son tantos los estudios que prestan atención al componente técnico de esta modalidad deportiva. De hecho, no hemos encontrado apenas documentos que contemplen las acciones que ejecuta el escalador en su ascenso considerando las dos extremidades del cuerpo -brazos y piernas- (De Benito, 2011), ya que en la escalada se ha dado mayor importancia a la acción de las extremidades superiores frente a las realizadas por las inferiores.

Podemos encontrar aportaciones como la de Muro, Vila, Vives y Gutiérrez (1994) quienes tras realizar un estudio antropométrico, biomecánico y fisiológico con cuatro escaladores de élite, establecieron una relación de los grupos musculares más importantes para la escalada: flexores del codo, flexores de muñeca, flexores de los dedos, flexores de cadera, extensores de rodilla y flexores plantares de tobillo. Por su parte, Peleteiro y García-López (2003) confeccionaron una revisión biomecánica de la distribución de las fuerzas aplicadas por el escalador en función de la inclinación de las paredes escaladas, de la cual concluyeron que el antebrazo era la masa muscular sometida a mayores solicitaciones. Sin embargo, ninguno de los dos estudios citados anteriormente hace referencia al tipo de activación muscular producida por el escalador a lo largo del ascenso.

Por ello, nos propusimos efectuar un análisis detallado de los grupos musculares requeridos durante el ascenso de las vías de escalada, en función del tipo de movimiento y el tipo de activación generada. De esta manera, la información recogida podría ser utilizada posteriormente para elaborar programas de entrenamiento específicos para cada escalador, en función de su modo de escalar (dinámica vs. estática) y de los movimientos necesarios para ejecutar una vía propuesta como objetivo.

En esta investigación se cuenta con una muestra de 72 escaladores de alto nivel, participando en competición federada, con el fin de analizar las acciones realizadas y las variables que influyen en su ejecución, y así posteriormente poder elaborar un esquema de patrones conductuales a través del análisis secuencial de los datos.

El objetivo de esta investigación fue analizar los movimientos técnicos que se producen en la escalada deportiva de dificultad durante la competición masculina de alto nivel y determinar las implicaciones musculares que subyacen a dichos movimientos. 
De Benito, A.M.; Sedano, S.; Redondo, J.C.; Cuadrado, G. (2013). Análisis cualitativo de las implicaciones musculares de la escalada deportiva de alto nivel en competición. RICYDE. Rev. int. cienc. deporte. 32(9), 154180. http://dx.doi.org/10.5232/ricyde2013.03205

\section{Método}

\section{Participantes}

La muestra está compuesta por 72 varones seleccionados de forma aleatoria de entre los 241 escaladores participantes en las pruebas de Copa de España de Escalada de Dificultad de 2009 y 2010. Dichos sujetos, expresaron tener un nivel técnico medio comprendido entre $7 \mathrm{C}^{+} / 8 \mathrm{~A}$ y $8 \mathrm{~A} / 8 \mathrm{~A}^{+}$para las categorías de nivel a vista y nivel trabajado respectivamente. Las características de la muestra se detallan a continuación (tabla 1).

Tabla 1: Características de los sujetos que componen la muestra

\begin{tabular}{|l|c|c|c|c|c|}
\hline & N & Mín. & Máx. & Media & SD \\
\hline Talla (cm) & 72 & 158 & 189 & 173,53 & 6,29 \\
\hline Envergadura (cm) & 72 & 158 & 208 & 176,99 & 10,65 \\
\hline Nivel técnico “a vista” & 72 & $7 \mathrm{~B}$ & $8 \mathrm{C}$ & $7 \mathrm{C}^{+} / 8 \mathrm{~A}$ & \\
\hline Nivel técnico “trabajado” & 72 & $7 \mathrm{C}$ & $9 \mathrm{~A}^{+}$ & $8 \mathrm{~A} / 8 \mathrm{~A}^{+}$ & \\
\hline
\end{tabular}

SD: Desviación estándar.

Las características de las vías analizadas fueron las siguientes: el nivel estimado de dificultad fue de $8 \mathrm{~A} / 8 \mathrm{~A}^{+}$para las vías clasificatorias y $8 \mathrm{~A}^{+} / 8 \mathrm{~B}$ para las vías finales; la inclinación media de ambos tipos fue de 112,6 \pm 3,65 ${ }^{\circ}$ respecto a la horizontal (según estudio de Couceiro, 2010), y las dimensiones del rocódromo artificial (conforme a la normativa de competición) fueron 12 metros de altura y 3 metros de anchura de vía, lo que permitió el montaje de vías con una longitud mínima de 15 metros. Además, la instalación contaba con un desplome en la parte superior del mismo (figura 1).

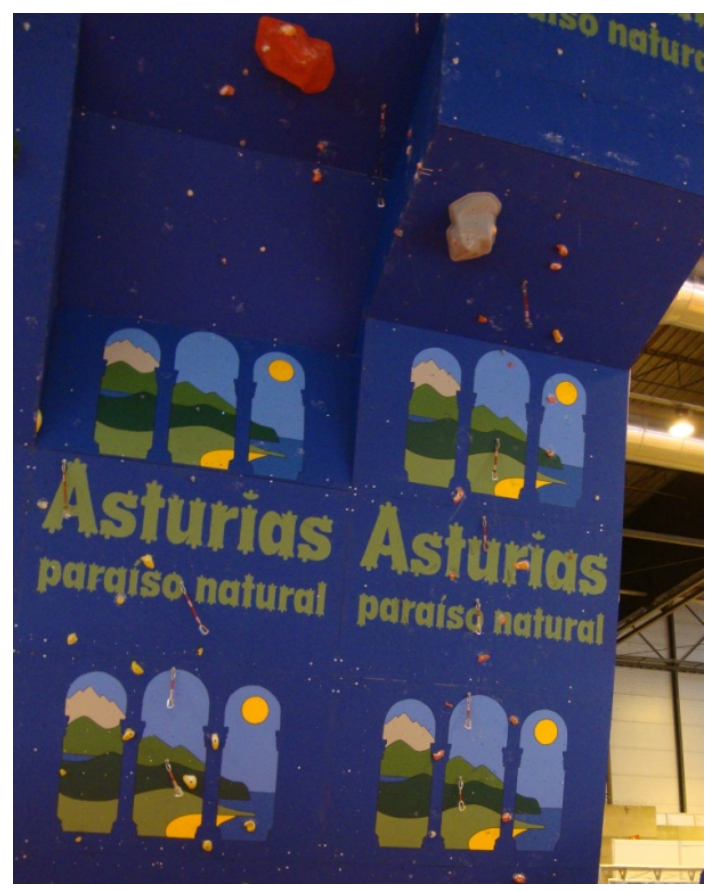

Figura1: Instalación artificial en donde se emplazaron las vías de escalada analizadas

Se registraron un total de 13.960 acciones, sin embargo no todas ellas forman parte de la muestra final. Se decidió que únicamente las acciones completas serían consideradas parte del estudio. Por ese motivo, la muestra del mismo resultó acotada en 7.738 movimientos, descritos claramente por su inicio y su final. 
De Benito, A.M.; Sedano, S.; Redondo, J.C.; Cuadrado, G. (2013). Análisis cualitativo de las implicaciones musculares de la escalada deportiva de alto nivel en competición. RICYDE. Rev. int. cienc. deporte. 32(9), 154180. http://dx.doi.org/10.5232/ricyde2013.03205

\section{Instrumentos}

La grabación de los videos fue realizada a través de una videocámara digital SONY ${ }^{\circledR}$, Handycam $^{\circledR}$ DCR-SR37E. Se utilizó el programa SPSS Statistics 19.0 para Windows ${ }^{\circledR}$, para verificar la calidad del dato y obtener la estadística descriptiva de los mismos.

\section{Metodología}

La elaboración y validación de la propuesta metodológica con la que observar, catalogar y posteriormente analizar los movimientos que ejecutan los escaladores a lo largo del ascenso fue motivo de un estudio previo (De Benito, García-Tormo, Izquierdo, Sedano, Redondo y Cuadrado, 2011) y se encuentra inserto dentro de la Metodología Observacional.

A continuación, se explican brevemente los niveles de respuesta utilizados en el estudio:

1. Acción (ACC): "movimiento global que suponga un desplazamiento del centro de gravedad del escalador o aquél que contemple el cambio de posición de cualquiera de las extremidades del mismo".

2. Posición corporal (PC): "posición del tronco del escalador durante el ascenso de la vía respecto a la horizontal”. Puede ser: vertical -próxima a los 90- (PC9), inclinada próxima a los $45^{\circ}$ - (PC4) o tumbada -próxima a los $0^{\circ}$ - (PC0).

3. Extremidad (E): “parte del cuerpo con que se está ejecutando la acción registrada”. Puede ser: extremidad superior o extremidad inferior.

4. Tiempo: revela el tiempo total que el escalador ha empleado en su ascenso. Para realizar el posterior análisis, las categorías de este nivel de respuesta se han agrupado en conjuntos de minuto, estableciéndose así un subnivel de respuesta “tiempo agrupado” (TAG), con cuatro subcategorías: tiempo agrupado uno (TAG1) -duración menor de dos minutos-, tiempo agrupado dos (TAG2) -duración comprendida entre dos y tres minutos-, tiempo agrupado tres (TAG3) -duración comprendida entre tres y cuatro minutos- y tiempo agrupado cuatro (TAG4) -duración mayor de cuatro minutos-.

5. Zona temporal (ZT): establece el momento en que se ejecutan las acciones. Existen seis categorías: zona temporal cero (ZT0) -acciones realizadas dentro del primer minuto-, zona temporal uno (ZT1) -acciones realizadas entre el primer y el segundo minuto-, zona temporal dos (ZT2) -acciones realizadas entre el segundo y el tercer minuto-, zona temporal tres (ZT3) -acciones realizadas entre el tercer y el cuarto minuto-, zona temporal cuatro (ZT4) -acciones realizadas entre el cuarto y el quinto minuto- y zona temporal cinco (ZT5) -acciones realizadas del quinto minuto en adelante-.

6. Inicio de movimiento (INI): contempla la posición origen de la extremidad que va a realizar el movimiento, respecto a la colocación del tronco. Existen 16 categorías: 1D, 2D, 3D, 4D, 5D, 6D, 7D, 8D, 1I, 2I, 3I, 4I, 5I, 6I, 7I, 8I.

7. Fin de movimiento (FIN): registra la posición final de la extremidad que ha realizado el movimiento, respecto a la colocación del tronco. Existen 16 categorías: 1D, 2D, 3D, 4D, 5D, 6D, 7D, 8D, 1I, 2I, 3I, 4I, 5I, 6I, 7I, 8I.

8. Movimiento (MOV): "acción en la que el segmento ejecutante (sea pie o mano) no abandona la presa o el lugar de impulso a partir del cual el escalador realiza su progresión y que conlleva, por tanto, algún tipo de contracción muscular efectiva”. Puede ser: tracción (TR), bloqueo (B), empuje (E), destrepe (D), progresión (P), equilibrio (EQ), talonamiento $(\mathrm{T})$ y destrepe inferior $(\mathrm{Di})$. 
De Benito, A.M.; Sedano, S.; Redondo, J.C.; Cuadrado, G. (2013). Análisis cualitativo de las implicaciones musculares de la escalada deportiva de alto nivel en competición. RICYDE. Rev. int. cienc. deporte. 32(9), 154180. http://dx.doi.org/10.5232/ricyde2013.03205

Los niveles de respuesta INI y FIN vienen definidos a través de un sistema de 16 cuadrantes, con referencias anatómicas, que dividen al cuerpo en hemisferio derecho e izquierdo, parte superior e inferior y cuadrantes proximales y distales respecto al eje longitudinal medial del cuerpo (De Benito y col., 2011) (figura 2).

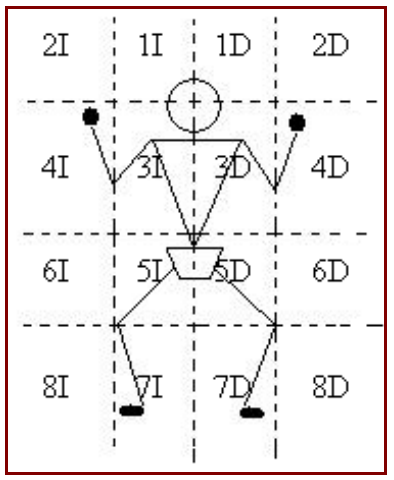

Figura 2: Sistema de cuadrantes utilizado para el sistema de categorías (De Benito y col., 2011)

El nivel de respuesta MOV surge de la combinación de los niveles de respuesta anteriores. Se subdivide en 8 subniveles de respuesta: “tracción” (TR), “bloqueo” (B), “empuje” (E) y “destrepe” (D) referentes a la extremidad superior y "progresión” (P), “equilibrio” (EQ), “talonamiento” (T) y “destrepe inferior” (Di) relativos a la extremidad inferior. A su vez, cada uno de los subniveles de respuesta, obtiene 4 subcategorías: "corto" (C), "corto cruzado" (CX), "largo" (L) y "largo cruzado" (LX), establecidas en función de la posición de inicio del segmento ejecutante y la trayectoria realizada (De Benito y col., 2011).

\section{Procedimiento}

Las pruebas de Copa de España fueron filmadas mediante una cámara de vídeo digital colocada conforme al protocolo establecido por De Benito y col. (2011). Posteriormente, las grabaciones fueron visionadas por cinco observadores externos que, de forma ciega e independiente, extrajeron los datos a una planilla de observación creada ad hoc para el estudio. Los observadores fueron seleccionados tras un periodo de entrenamiento y de control de calidad del dato, con el fin de asegurar la corrección de los datos registrados y evitar los posibles sesgos.

\section{Análisis de datos}

El proceso de verificación de la calidad del dato se dividió en dos partes: la concordancia interobservador y la concordancia intraobservador, evaluándose la fiabilidad existente entre los observadores, a partir de la comparación de un modelo y la fiabilidad de cada uno de los observadores, a partir de un doble visionado del mismo ascenso.

El posterior análisis de los datos se llevó a cabo a través del programa informático SPSS 19.0 para Windows ${ }^{\circledR}$ con el que se calcularon el coeficiente kappa de Cohen y los estadísticos descriptivos: frecuencias y porcentajes. 
De Benito, A.M.; Sedano, S.; Redondo, J.C.; Cuadrado, G. (2013). Análisis cualitativo de las implicaciones musculares de la escalada deportiva de alto nivel en competición. RICYDE. Rev. int. cienc. deporte. 32(9), 154180. http://dx.doi.org/10.5232/ricyde2013.03205

\section{Análisis de fiabilidad}

La fiabilidad de los datos recogidos fue evaluada a través del análisis de concordancia interobservador e intraobservador, utilizado el coeficiente kappa de Cohen para cada uno de los niveles de respuesta registrados.

Para evaluar la concordancia interobservador se compararon los datos obtenidos de cada uno de los observadores con el modelo de referencia del observador principal, el cual realizó el visionado del mismo ascenso por duplicado obteniendo una fiabilidad del 99\%, por lo que se toman sus registros como modelo comparativo para el resto de observadores.

Por otra parte, para obtener el nivel de concordancia intraobservador se compararon los datos registrados por los observadores en dos visionados de la misma prueba con 10 días de descanso entre ambos análisis (Medina y Delgado, 1999).

A continuación se presenta la tabla 2 en la que aparecen reflejados los resultados obtenidos en cada nivel de respuesta respecto a la fiabilidad interobservador e intraobservador respectivamente, así como también el valor promedio de cada observador. Como se puede observar, los resultados obtenidos en los niveles de respuesta analizados superan el mínimo establecido por Cohen (1960) de 0,610 siendo 0,905 el valor medio de fiabilidad del estudio.

Tabla 2: Valores de los índices de kappa de Cohen en los diferentes niveles de respuesta

\begin{tabular}{|l|c|c|}
\hline & $\begin{array}{c}\text { Interobservador } \\
\text { Modelo y Obs (1-5) }\end{array}$ & $\begin{array}{c}\text { Intraobservador } \\
\text { Obs1 }^{1} \text { y Obs1 }\end{array}$ \\
\hline Acción & 1,000 & 1,000 \\
\hline Posición corporal & 0,917 & 0,964 \\
\hline Extremidad & 1,000 & 1,000 \\
\hline Tiempo & 0,892 & 0,900 \\
\hline Inicio & 0,849 & 0,794 \\
\hline Fin & 0,795 & 0,736 \\
\hline Total & 0,909 & 0,899 \\
\cline { 2 - 3 } & \multicolumn{2}{|c|}{0,904} \\
\hline
\end{tabular}

Obs.: Observador; Obs1 ${ }^{1}$ : primera observación; Obs1 ${ }^{2}$ : segunda observación.

\section{Resultados}

\section{Análisis descriptivo}

La tabla 3 muestra los resultados obtenidos en función del nivel de respuesta "posición corporal”, variable que refleja la posición que adopta el tronco del escalador respecto a la horizontal, durante el ascenso de la vía.

Tabla 3: Registro de los movimientos que integran la muestra en función de la inclinación de la vía

\begin{tabular}{|l|c|c|}
\hline & \multicolumn{2}{|c|}{ Posición corporal } \\
\hline & Frecuencia & Porcentaje \\
\hline PC0 & 1.396 & $18,0 \%$ \\
\hline PC4 & 5.586 & $72,2 \%$ \\
\hline PC9 & 756 & $9,8 \%$ \\
\hline Total & 7.738 & $100,0 \%$ \\
\hline
\end{tabular}

PC0: tumbado; PC4: inclinado; PC9: vertical. 
De Benito, A.M.; Sedano, S.; Redondo, J.C.; Cuadrado, G. (2013). Análisis cualitativo de las implicaciones musculares de la escalada deportiva de alto nivel en competición. RICYDE. Rev. int. cienc. deporte. 32(9), 154180. http://dx.doi.org/10.5232/ricyde2013.03205

Los resultados respecto al nivel de respuesta “posición corporal”, revelan que el 72,2\% de los movimientos analizados se realizaron en posición inclinada (PC4), mientras que el 18,0\% se ejecutaron en posición tumbada (PC0) y el 9,8\% restante, en posición vertical (PC9).

La tabla 4 ofrece los resultados obtenidos respecto al nivel de respuesta "extremidad". Se puede observar cómo los movimientos ejecutados por la extremidad superior suponen el $62,69 \%$ de las acciones, mientras que el 37,31\% son efectuadas por la extremidad inferior.

Tabla 4: Distribución de los movimientos de la muestra según la extremidad

\begin{tabular}{|l|c|c|}
\hline & \multicolumn{2}{|c|}{ Extremidad } \\
\hline & Frecuencia & Porcentaje \\
\hline Extremidad superior & 4.851 & $62,69 \%$ \\
\hline Extremidad inferior & 2.887 & $37,31 \%$ \\
\hline Total & 7.738 & $100,0 \%$ \\
\hline
\end{tabular}

Puesto que los resultados encontrados podrían estar condicionados por la inclinación de las vías que componen la muestra y por tanto por la posición corporal que adopta el sujeto en su ascenso, la tabla 5 muestra los resultados obtenidos respecto a la asociación entre ambos niveles de respuesta: "posición corporal” y "extremidad”.

Tabla 5: Distribución de los movimientos según la posición corporal y la extremidad ejecutante

\begin{tabular}{|c|c|c|c|c|c|}
\hline & \multicolumn{2}{|c|}{ Extremidad superior } & \multicolumn{2}{c|}{ Extremidad inferior } & \\
\hline & $\mathrm{N}$ & $\%$ & $\mathrm{~N}$ & $\%$ & Total \\
\hline PC0 & 884 & $63,32 \%$ & 512 & $36,68 \%$ & 1396 \\
\hline PC4 & 3533 & $63,25 \%$ & 2053 & $36,75 \%$ & 5586 \\
\hline PC9 & 434 & $57,41 \%$ & 322 & $42,59 \%$ & 756 \\
\hline
\end{tabular}

PC0: tumbado; PC4: inclinado; PC9: vertical.

En la tabla 6 se recogen los datos analizados en función del subnivel de respuesta "tiempo agrupado". Se obtienen porcentajes similares entre los grupos TAG1 y TAG2 con 12,20\% y 15,39\% respectivamente, mientras que los grupos TAG3 y TAG4 muestran porcentajes más elevados: 33,92\% y 38,49\%, respectivamente.

Tabla 6: Distribución de los movimientos en función del tiempo agrupado

\begin{tabular}{|l|c|c|}
\hline & \multicolumn{2}{|c|}{ Tiempo agrupado } \\
\hline & Frecuencia & Porcentaje \\
\hline TAG1 (<2') & 944 & $12,20 \%$ \\
\hline TAG2 (2-3') & 1191 & $15,39 \%$ \\
\hline TAG3 (3-4') & 2625 & $33,92 \%$ \\
\hline TAG4 ( $\left.>4^{\prime}\right)$ & 2978 & $38,49 \%$ \\
\hline Total & 7738 & $100,0 \%$ \\
\hline
\end{tabular}

TAG1: tiempo agrupado 1; TAG2: tiempo agrupado 2; TAG3: tiempo agrupado 3; TAG4: tiempo agrupado 4.

En la tabla 7 se ofrecen los resultados obtenidos respecto al nivel de respuesta "zona tiempo". Los datos muestran la existencia de una relación inversa entre el tiempo transcurrido y el número de acciones registradas. El mayor número de movimientos observados se producen en el primer minuto de duración, con un porcentaje de 36,61\%, hasta llegar al rango más bajo que muestra sólo un $0,34 \%$ y que corresponde a aquellos movimientos que se ejecutaron sobrepasados los cinco minutos. 
De Benito, A.M.; Sedano, S.; Redondo, J.C.; Cuadrado, G. (2013). Análisis cualitativo de las implicaciones musculares de la escalada deportiva de alto nivel en competición. RICYDE. Rev. int. cienc. deporte. 32(9), 154180. http://dx.doi.org/10.5232/ricyde2013.03205

Tabla 7: Distribución de los movimientos en función de la zona tiempo

\begin{tabular}{|l|c|c|}
\hline & \multicolumn{2}{|c|}{ Zona tiempo } \\
\hline & Frecuencia & Porcentaje \\
\hline ZT0 (<1') & 2833 & $36,61 \%$ \\
\hline ZT1 (1-2') & 2161 & $27,93 \%$ \\
\hline ZT2 (2-3') & 1376 & $17,78 \%$ \\
\hline ZT3 (3-4') & 900 & $11,63 \%$ \\
\hline ZT4 (4-5') & 442 & $5,71 \%$ \\
\hline ZT5 ( $\left.>5^{\prime}\right)$ & 26 & $0,34 \%$ \\
\hline Total & 7738 & $100,0 \%$ \\
\hline
\end{tabular}

ZT0: zona tiempo 0; ZT1: zona tiempo 1; ZT2: zona tiempo 2; ZT3: zona tiempo 3; ZT4: zona tiempo 4; ZT5: zona tiempo 5 .

La tabla 8 ofrece la distribución de los movimientos registrados de la extremidad superior e inferior, conforme a sus subniveles de respuesta: "tracción” (TR), "bloqueo” (B), “empuje” (E), “destrepe” (D), “progresión” (P), “equilibrio” (EQ), “talonamiento” (T) y “destrepe inferior” (Di). En la extremidad superior, el mayor porcentaje de las acciones registradas corresponde al subnivel B con un 50,86\%. Por su parte, en la extremidad inferior, el mayor porcentaje de las acciones registradas corresponde al subnivel EQ con un 58,26\%.

Tabla 8: Distribución del nivel de respuesta movimiento

\begin{tabular}{|c|c|c|c|c|c|c|c|}
\hline \multicolumn{3}{|c|}{ Extremidad Superior } & \multicolumn{4}{c|}{ Extremidad Inferior } \\
\hline TR & B & E & D & P & EQ & T & Di \\
\hline $35,31 \%$ & $50,86 \%$ & $11,87 \%$ & $1,96 \%$ & $34,92 \%$ & $58,26 \%$ & $5,16 \%$ & $1,66 \%$ \\
\hline
\end{tabular}

TR: Tracción; B: Bloqueo; E: Empuje; D: Destrepe; P: Progresión; EQ: Equilibrio; T: Talonamiento; Di: Destrepe inferior.

Puesto que los resultados encontrados podrían estar condicionados de nuevo por la posición corporal que adopta el sujeto en su ascenso, la tabla 9 muestra los resultados obtenidos respecto a la asociación entre los subniveles de respuesta de la extremidad superior e inferior y la "posición corporal”.

Tabla 9: Asociación subniveles de respuesta de la extremidad superior y posición corporal

\begin{tabular}{|c|c|c|c|c|c|c|c|c|}
\hline & \multicolumn{3}{|c|}{ Extremidad Superior } & \multicolumn{4}{c|}{ Extremidad Inferior } \\
\hline & TR & B & E & D & P & EQ & T & Di \\
\hline PC0 & $26,7 \%$ & $58,03 \%$ & $12,56 \%$ & $2,71 \%$ & $23,24 \%$ & $66,80 \%$ & $8,20 \%$ & $1,76 \%$ \\
\hline PC4 & $37,5 \%$ & $49,28 \%$ & $11,80 \%$ & $1,42 \%$ & $40,04 \%$ & $54,21 \%$ & $4,14 \%$ & $6,83 \%$ \\
\hline PC9 & $35,02 \%$ & $49,08 \%$ & $11,06 \%$ & $4,84 \%$ & $20,81 \%$ & $70,50 \%$ & $1,61 \%$ & $1,86 \%$ \\
\hline
\end{tabular}

PC0: tumbado; PC4: inclinado; PC9: vertical; TR: Tracción; B: Bloqueo; E: Empuje; D: Destrepe; P: Progresión; EQ: Equilibrio; T: Talonamiento; Di: Destrepe inferior.

Las tablas 10 y 11 muestran los resultados obtenidos en función de los distintos subniveles de respuesta, de la extremidad superior e inferior, respectivamente.

Se hace patente el hecho de que las acciones cortas, TRC, BC, ECX, DC realizadas por la extremidad superior y PC, EQC y DiC realizadas por la extremidad inferior, suponen los mayores porcentajes de la muestra, con valores comprendidos entre los 45,83\% y 80,08\%. 
De Benito, A.M.; Sedano, S.; Redondo, J.C.; Cuadrado, G. (2013). Análisis cualitativo de las implicaciones musculares de la escalada deportiva de alto nivel en competición. RICYDE. Rev. int. cienc. deporte. 32(9), 154180. http://dx.doi.org/10.5232/ricyde2013.03205

Tabla 10: Distribución de las subcategorías de la extremidad superior

\begin{tabular}{|c|c|c|c|c|}
\hline & \multicolumn{4}{|c|}{ Extremidad Superior } \\
\hline & C & CX & L & LX \\
\hline TR & $53,6 \%$ & $8,2 \%$ & $10,7 \%$ & $27,5 \%$ \\
\hline B & $76,9 \%$ & $2,4 \%$ & $20,6 \%$ & $0,0 \%$ \\
\hline E & $16,3 \%$ & $79,5 \%$ & $3,1 \%$ & $1,0 \%$ \\
\hline D & $53,7 \%$ & $14,7 \%$ & $21,0 \%$ & $10,5 \%$ \\
\hline
\end{tabular}

TR: Tracción; B: Bloqueo; E: Empuje; D: Destrepe; C: Corto; CX: Corto cruzado; L: Largo; LX: Largo cruzado.

Tabla 11: Distribución de las subcategorías de la extremidad inferior

\begin{tabular}{|c|c|c|c|c|}
\hline & \multicolumn{4}{|c|}{ Extremidad Inferior } \\
\hline & $\mathrm{C}$ & $\mathrm{CX}$ & $\mathrm{L}$ & $\mathrm{LX}$ \\
\hline $\mathrm{P}$ & $59,6 \%$ & $18,6 \%$ & $11,1 \%$ & $10,6 \%$ \\
\hline $\mathrm{EQ}$ & $80,1 \%$ & $4,2 \%$ & $15,6 \%$ & $0,0 \%$ \\
\hline $\mathrm{T}$ & $14,8 \%$ & $8,0 \%$ & $24,8 \%$ & $52,3 \%$ \\
\hline $\mathrm{Di}$ & $45,8 \%$ & $12,5 \%$ & $29,2 \%$ & $12,5 \%$ \\
\hline
\end{tabular}

P: Progresión; EQ: Equilibrio; T: Talonamiento; Di: Destrepe inferior; C: Corto; CX: Corto cruzado; L: Largo; LX: Largo cruzado.

En el apartado introductorio, se hizo referencia al hecho de que la importancia de este estudio radica en el intento de realizar un análisis minucioso de cada uno de los movimientos que ejecutan los escaladores. Se presentan por ello a continuación, las tablas del número 12 al 22, mostrando el análisis de las subcategorías de movimiento, en función de la implicación muscular que subyace a su ejecución. Se detallan las acciones articulares, los grupos musculares responsables de dichos movimientos y el tipo de contracción generada, así como también, el porcentaje de la distribución de los diferentes movimientos respecto al total.

La información aportada por dichas tablas, permite establecer la relación de los grupos musculares con mayor participación en los movimientos de la escalada, así como también el modo en que estos actúan. 
De Benito, A.M.; Sedano, S.; Redondo, J.C.; Cuadrado, G. (2013). Análisis cualitativo de las implicaciones musculares de la escalada deportiva de alto nivel en competición. RICYDE. Rev. int. cienc. deporte. 32(9), 154-180. http://dx.doi.org/10.5232/ricyde2013.03205

La tabla 12 presenta el análisis de las implicaciones musculares que subyacen a los movimientos de tracción realizados por la extremidad superior y respecto a los grupos musculares que actúan respecto a las articulaciones del hombro y el codo.

Tabla 12: Implicación muscular del movimiento de “Tracción” de la extremidad superior

\begin{tabular}{|c|c|c|c|c|c|c|c|c|c|}
\hline & & Inic & Fin & & HOMBRO & & & CODO & \\
\hline $\begin{array}{c}\text { SUBNIVEL } \\
\text { DE } \\
\text { RESPUESTA }\end{array}$ & CÓDIGO & $\begin{array}{l}\text { Mano } \\
\text { Dcha. }\end{array}$ & $\begin{array}{l}\text { Mano } \\
\text { Izda. }\end{array}$ & Acción & $\begin{array}{c}\text { Grupos } \\
\text { musculares }\end{array}$ & $\begin{array}{c}\text { Tipo } \\
\text { Contracción }\end{array}$ & Acción & $\begin{array}{c}\text { Grupos } \\
\text { musculares }\end{array}$ & $\begin{array}{c}\text { Tipo } \\
\text { Contracción }\end{array}$ \\
\hline \multirow{15}{*}{ 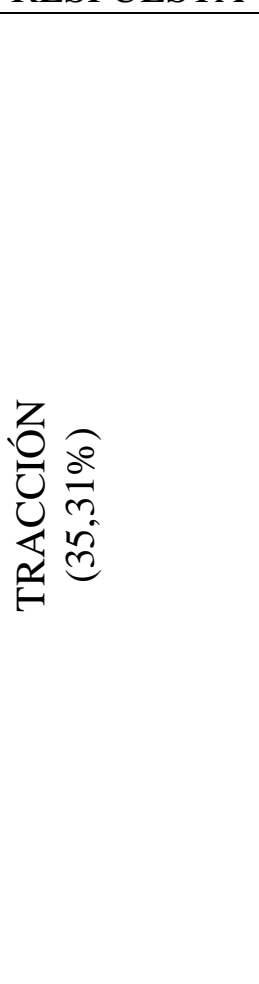 } & \multirow{2}{*}{$\begin{array}{c}\text { TRC } \\
(53,6 \%)\end{array}$} & \multirow[t]{2}{*}{ 1D3D } & \multirow[t]{2}{*}{ 1I3I } & \multirow[t]{2}{*}{ Extensión } & \multirow[t]{2}{*}{ Extensores } & \multirow[t]{2}{*}{ Concéntrica } & \multirow{2}{*}{$\begin{array}{l}\text { Flexión en } \\
\text { pronación }\end{array}$} & Flexores & Concéntrica \\
\hline & & & & & & & & Pronadores & Concéntrica \\
\hline & \multirow[t]{5}{*}{$\begin{array}{l}\text { TRCX } \\
(8,2 \%)\end{array}$} & 1D1I & 1I1D & $\begin{array}{c}\text { Flexión } \\
\text { horizontal }\end{array}$ & $\begin{array}{c}\text { Flexores } \\
\text { horizontales }\end{array}$ & Concéntrica & No mov. & Flexores & Isométrica \\
\hline & & \multirow[t]{2}{*}{ 1D3I } & \multirow[t]{2}{*}{ 1I3D } & Extensión & Extensores & Concéntrica & \multirow{2}{*}{$\begin{array}{l}\text { Flexión en } \\
\text { pronación }\end{array}$} & Flexores & Concéntrica \\
\hline & & & & $\begin{array}{c}\text { Flexión } \\
\text { horizontal }\end{array}$ & $\begin{array}{c}\text { Flexores } \\
\text { horizontales }\end{array}$ & Concéntrica & & Pronadores & Concéntrica \\
\hline & & \multirow[t]{2}{*}{ 1I3I } & \multirow[t]{2}{*}{ 1D3D } & \multirow[t]{2}{*}{ Extensión } & \multirow[t]{2}{*}{ Extensores } & \multirow[t]{2}{*}{ Concéntrica } & \multirow{2}{*}{$\begin{array}{l}\text { Flexión en } \\
\text { pronación }\end{array}$} & Flexores & Concéntrica \\
\hline & & & & & & & & Pronadores & Concéntrica \\
\hline & \multirow{2}{*}{$\begin{array}{c}\text { TRL } \\
(10,7 \%)\end{array}$} & \multirow[t]{2}{*}{ 2D4D } & \multirow[t]{2}{*}{ 2I4I } & Extensión & Extensores & Concéntrica & \multirow[t]{2}{*}{ No mov. } & \multirow[t]{2}{*}{ Supinadores } & \multirow[t]{2}{*}{ Isométrica } \\
\hline & & & & Aducción & Aductores & Concéntrica & & & \\
\hline & \multirow{6}{*}{$\begin{array}{c}\text { TRLX } \\
(27,5 \%)\end{array}$} & \multirow[t]{2}{*}{ 4D3D } & \multirow[t]{2}{*}{ 4I3I } & Extensión & Extensores & Concéntrica & \multirow{2}{*}{$\begin{array}{l}\text { Flexión en } \\
\text { pronación }\end{array}$} & Flexores & Concéntrica \\
\hline & & & & Aducción & Aductores & Concéntrica & & Pronadores & Concéntrica \\
\hline & & \multirow[t]{2}{*}{ 2D1D } & \multirow[t]{2}{*}{ 2I1I } & \multirow{2}{*}{$\begin{array}{c}\text { Flexión } \\
\text { horizontal }\end{array}$} & \multirow{2}{*}{$\begin{array}{c}\text { Flexores } \\
\text { horizontales }\end{array}$} & \multirow[t]{2}{*}{ Concéntrica } & \multirow{2}{*}{$\begin{array}{l}\text { Flexión en } \\
\text { pronación }\end{array}$} & Flexores & Concéntrica \\
\hline & & & & & & & & Pronadores & Concéntrica \\
\hline & & \multirow[t]{2}{*}{ 2D3D } & \multirow[t]{2}{*}{ 2I3I } & Extensión & Extensores & Concéntrica & Flexión en & Flexores & Concéntrica \\
\hline & & & & Aducción & Aductores & Concéntrica & pronación & Pronadores & Concéntrica \\
\hline
\end{tabular}


De Benito, A.M.; Sedano, S.; Redondo, J.C.; Cuadrado, G. (2013). Análisis cualitativo de las implicaciones musculares de la escalada deportiva de alto nivel en competición. RICYDE. Rev. int. cienc. deporte. 32(9), 154-180. http://dx.doi.org/10.5232/ricyde2013.03205

La tabla 13 presenta el análisis de las implicaciones musculares que subyacen a los movimientos de bloqueo realizados por la extremidad superior y respecto a los grupos musculares que actúan respecto a las articulaciones del hombro y el codo.

Tabla 13: Implicación muscular del movimiento de “Bloqueo” de la extremidad superior

\begin{tabular}{|c|c|c|c|c|c|c|c|c|c|}
\hline & & & Fin & & HOMBRO & & & CODO & \\
\hline $\begin{array}{c}\text { SUBNIVEL } \\
\text { DE } \\
\text { RESPUESTA }\end{array}$ & CÓDIGO & $\begin{array}{l}\text { Mano } \\
\text { Dcha. }\end{array}$ & $\begin{array}{l}\text { Mano } \\
\text { Izda. }\end{array}$ & Acción & $\begin{array}{c}\text { Grupos } \\
\text { musculares }\end{array}$ & $\begin{array}{c}\text { Tipo } \\
\text { Contracción }\end{array}$ & Acción & $\begin{array}{c}\text { Grupos } \\
\text { musculares }\end{array}$ & $\begin{array}{c}\text { Tipo } \\
\text { Contracción }\end{array}$ \\
\hline \multirow{12}{*}{ 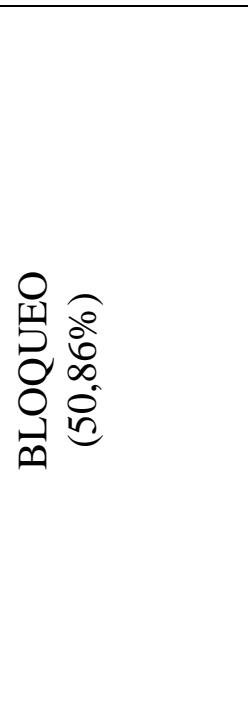 } & \multirow{2}{*}{$\begin{array}{c}\text { BC } \\
(76,9 \%)\end{array}$} & 1D1D & 1I1I & No mov. & Extensores & Isométrica & No mov. & Flexores & Isométrica \\
\hline & & 3D3D & 3I3I & No mov. & Extensores & Isométrica & No mov. & Flexores & Isométrica \\
\hline & \multirow{4}{*}{$\begin{array}{c}\text { BCX } \\
(2,4 \%)\end{array}$} & \multirow[t]{2}{*}{ 1I1I } & \multirow[t]{2}{*}{ 1D1D } & No mov. & Extensores & Isométrica & \multirow[t]{2}{*}{ No mov. } & \multirow[t]{2}{*}{ Flexores } & \multirow[t]{2}{*}{ Isométrica } \\
\hline & & & & No mov. & Abductores & Isométrica & & & \\
\hline & & \multirow[t]{2}{*}{ 3I3I } & \multirow[t]{2}{*}{ 3D3D } & No mov. & Extensores & Isométrica & \multirow[t]{2}{*}{ No mov. } & \multirow[t]{2}{*}{ Flexores } & \multirow[t]{2}{*}{ Isométrica } \\
\hline & & & & No mov. & Abductores & Isométrica & & & \\
\hline & \multirow{2}{*}{$\begin{array}{c}\text { BL } \\
(20,6 \%)\end{array}$} & 2D2D & 2I2I & No mov. & Aductores & Isométrica & No mov. & Flexores & Isométrica \\
\hline & & 4D4D & $4 \mathrm{I} 4 \mathrm{I}$ & No mov. & Aductores & Isométrica & No mov. & Flexores & Isométrica \\
\hline & \multirow{4}{*}{$\begin{array}{c}\text { BLX } \\
(0,0 \%)\end{array}$} & \multirow[t]{2}{*}{ 2I2I } & \multirow[t]{2}{*}{ 2D2D } & \multirow[t]{2}{*}{ No mov. } & \multirow{2}{*}{$\begin{array}{c}\text { Extensores } \\
\text { horizontales }\end{array}$} & \multirow[t]{2}{*}{ Isométrica } & No mov. & Flexores & Isométrica \\
\hline & & & & & & & No mov. & Pronadores & Isométrica \\
\hline & & \multirow[t]{2}{*}{ 4I4I } & \multirow[t]{2}{*}{ 4D4D } & \multirow[t]{2}{*}{ No mov. } & \multirow{2}{*}{$\begin{array}{c}\text { Extensores } \\
\text { horizontales }\end{array}$} & \multirow[t]{2}{*}{ Isométrica } & No mov. & Flexores & Isométrica \\
\hline & & & & & & & No mov. & Pronadores & Isométrica \\
\hline
\end{tabular}


De Benito, A.M.; Sedano, S.; Redondo, J.C.; Cuadrado, G. (2013). Análisis cualitativo de las implicaciones musculares de la escalada deportiva de alto nivel en competición. RICYDE. Rev. int. cienc. deporte. 32(9), 154-180. http://dx.doi.org/10.5232/ricyde2013.03205

Las tablas 14 y 15 presentan el análisis de las implicaciones musculares que subyacen a los movimientos de empuje realizados por la extremidad superior y respecto a los grupos musculares que actúan respecto a las articulaciones del hombro y el codo.

Tabla 14: Implicación muscular del movimiento de "Empuje” de la extremidad superior

\begin{tabular}{|c|c|c|c|c|c|c|c|c|c|}
\hline & & & Fin & & HOMBRO & & & CODO & \\
\hline $\begin{array}{c}\text { SUBNIVEL } \\
\text { DE } \\
\text { RESPUESTA }\end{array}$ & CÓDIGO & $\begin{array}{l}\text { Mano } \\
\text { Dcha. }\end{array}$ & $\begin{array}{l}\text { Mano } \\
\text { Izda. }\end{array}$ & Acción & $\begin{array}{c}\text { Grupos } \\
\text { musculares }\end{array}$ & $\begin{array}{c}\text { Tipo } \\
\text { Contracción }\end{array}$ & Acción & $\begin{array}{c}\text { Grupos } \\
\text { musculares }\end{array}$ & $\begin{array}{c}\text { Tipo } \\
\text { Contracción }\end{array}$ \\
\hline \multirow{13}{*}{ 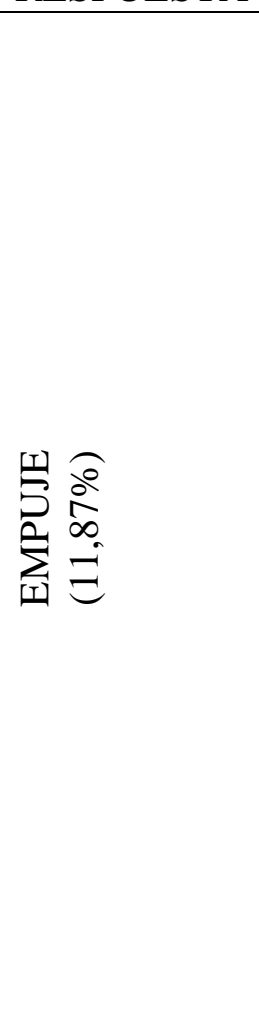 } & \multirow{2}{*}{$\begin{array}{c}\text { EC } \\
(16,3 \%)\end{array}$} & \multirow[t]{2}{*}{ 3D5D } & \multirow[t]{2}{*}{$3 I 5 I$} & \multirow[t]{2}{*}{ Extensión } & \multirow[t]{2}{*}{ Extensores } & \multirow[t]{2}{*}{ Concéntrica } & \multirow{2}{*}{$\begin{array}{l}\text { Extensión } \\
\text { en } \\
\text { pronación }\end{array}$} & Extensores & Concéntrica \\
\hline & & & & & & & & Pronadores & Concéntrica \\
\hline & \multirow{11}{*}{$\begin{array}{c}\text { ECX } \\
(79,5 \%)\end{array}$} & \multirow[t]{2}{*}{ 1D2D } & \multirow[t]{2}{*}{ 1I2I } & \multirow{2}{*}{$\begin{array}{l}\text { Extensión } \\
\text { horizontal }\end{array}$} & \multirow{2}{*}{$\begin{array}{l}\text { Extensores } \\
\text { horizontales }\end{array}$} & \multirow[t]{2}{*}{ Concéntrica } & \multirow{2}{*}{$\begin{array}{l}\text { Extensión } \\
\text { en } \\
\text { pronación }\end{array}$} & Extensores & Concéntrica \\
\hline & & & & & & & & Pronadores & Concéntrica \\
\hline & & \multirow[t]{3}{*}{ 1D4D } & \multirow[t]{3}{*}{ 1I4I } & Extensión & Extensores & Concéntrica & \multirow{3}{*}{$\begin{array}{l}\text { Flexión } \\
\text { en } \\
\text { pronación }\end{array}$} & \multirow[t]{2}{*}{ Flexores } & \multirow[t]{2}{*}{ Concéntrica } \\
\hline & & & & $\begin{array}{l}\text { Extensión } \\
\text { horizontal }\end{array}$ & $\begin{array}{l}\text { Extensores } \\
\text { horizontales }\end{array}$ & Concéntrica & & & \\
\hline & & & & $\begin{array}{l}\text { Rotación } \\
\text { externa }\end{array}$ & $\begin{array}{c}\text { Rotadores } \\
\text { externos }\end{array}$ & Concéntrica & & Pronadores & Concéntrica \\
\hline & & \multirow[t]{3}{*}{ 3D4D } & \multirow[t]{3}{*}{ 3I4I } & Extensión & Extensores & Concéntrica & \multirow{3}{*}{$\begin{array}{c}\text { Extensión } \\
\text { en } \\
\text { pronación }\end{array}$} & Extensores & Concéntrica \\
\hline & & & & horizontal & horizontales & & & Pronadores & Concéntrica \\
\hline & & & & $\begin{array}{c}\text { Rotación } \\
\text { externa }\end{array}$ & $\begin{array}{c}\text { Rotadores } \\
\text { externos }\end{array}$ & Concéntrica & & & \\
\hline & & \multirow[t]{3}{*}{ 3D6D } & \multirow[t]{3}{*}{ 3I6I } & Abducción & Abductores & Concéntrica & \multirow{3}{*}{$\begin{array}{l}\text { Extensión } \\
\text { en } \\
\text { pronación }\end{array}$} & Extensores & Concéntrica \\
\hline & & & & $\begin{array}{l}\text { Extensión } \\
\text { horizontal }\end{array}$ & $\begin{array}{l}\text { Extensores } \\
\text { horizontales }\end{array}$ & Concéntrica & & Pronadores & Concéntrica \\
\hline & & & & $\begin{array}{c}\text { Rotación } \\
\text { externa }\end{array}$ & $\begin{array}{c}\text { Rotadores } \\
\text { externos }\end{array}$ & Concéntrica & & & \\
\hline
\end{tabular}


De Benito, A.M.; Sedano, S.; Redondo, J.C.; Cuadrado, G. (2013). Análisis cualitativo de las implicaciones musculares de la escalada deportiva de alto nivel en competición. RICYDE. Rev. int. cienc. deporte. 32(9), 154-180. http://dx.doi.org/10.5232/ricyde2013.03205

Tabla 15: Implicación muscular del movimiento de “Empuje” de la extremidad superior (continuación de tabla 14)

\begin{tabular}{|c|c|c|c|c|c|c|c|c|c|}
\hline & & \multicolumn{2}{|c|}{ Inicio-Fin } & \multicolumn{3}{|c|}{ HOMBRO } & \multicolumn{3}{|c|}{ CODO } \\
\hline $\begin{array}{l}\text { SUBNIVEL } \\
\text { DE } \\
\text { RESPUESTA }\end{array}$ & CÓDIGO & $\begin{array}{l}\text { Mano } \\
\text { Dcha. }\end{array}$ & $\begin{array}{l}\text { Mano } \\
\text { Izda. }\end{array}$ & Acción & $\begin{array}{c}\text { Grupos } \\
\text { musculares }\end{array}$ & $\begin{array}{c}\text { Tipo } \\
\text { Contracción }\end{array}$ & Acción & $\begin{array}{c}\text { Grupos } \\
\text { musculares }\end{array}$ & $\begin{array}{c}\text { Tipo } \\
\text { Contracción }\end{array}$ \\
\hline \multirow{8}{*}{ 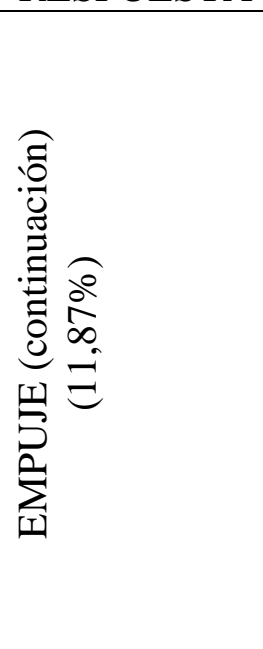 } & \multirow{4}{*}{$\begin{array}{c}\text { ECX } \\
(79,5 \%)\end{array}$} & \multirow[t]{2}{*}{ 3D3I } & \multirow[t]{2}{*}{ 3I3D } & Flexión & Flexores & Concéntrica & \multirow[t]{2}{*}{ No mov. } & \multirow[t]{2}{*}{ Flexores } & \multirow[t]{2}{*}{ Isométrica } \\
\hline & & & & Aducción & Aductores & Concéntrica & & & \\
\hline & & \multirow[t]{2}{*}{ 3D5I } & \multirow[t]{2}{*}{$3 I 5 D$} & Aducción & Aductores & Concéntrica & \multirow{2}{*}{$\begin{array}{l}\text { Extensión } \\
\text { en } \\
\text { pronación }\end{array}$} & Extensores & Concéntrica \\
\hline & & & & $\begin{array}{c}\text { Rotación } \\
\text { interna }\end{array}$ & $\begin{array}{l}\text { Rotadores } \\
\text { internos }\end{array}$ & Concéntrica & & Pronadores & Concéntrica \\
\hline & \multirow{2}{*}{$\begin{array}{c}\text { EL } \\
(3,1 \%)\end{array}$} & \multirow[t]{2}{*}{ 4D6D } & \multirow[t]{2}{*}{$4 \mathrm{I} 6 \mathrm{I}$} & Extensión & Extensores & Concéntrica & Extensión & Extensores & Concéntrica \\
\hline & & & & Aducción & Aductores & Concéntrica & Pronación & Pronadores & Concéntrica \\
\hline & \multirow[t]{2}{*}{$\begin{array}{c}\text { ELX } \\
(1,0 \%)\end{array}$} & \multirow[t]{2}{*}{ 4D5D } & \multirow[t]{2}{*}{$4 \mathrm{I} 5 \mathrm{I}$} & Aducción & Aductores & Concéntrica & $\begin{array}{c}\text { Flexión } \\
\text { en } \\
\text { pronación }\end{array}$ & Flexores & Concéntrica \\
\hline & & & & $\begin{array}{l}\text { Rotación } \\
\text { interna }\end{array}$ & $\begin{array}{l}\text { Rotadores } \\
\text { internos }\end{array}$ & Concéntrica & Pronación & Pronadores & Concéntrica \\
\hline
\end{tabular}


De Benito, A.M.; Sedano, S.; Redondo, J.C.; Cuadrado, G. (2013). Análisis cualitativo de las implicaciones musculares de la escalada deportiva de alto nivel en competición. RICYDE. Rev. int. cienc. deporte. 32(9), 154-180. http://dx.doi.org/10.5232/ricyde2013.03205

La tabla 16 presenta el análisis de las implicaciones musculares que subyacen a los movimientos de destrepe realizados por la extremidad superior y respecto a los grupos musculares que actúan respecto a las articulaciones del hombro y el codo.

Tabla 16: Implicación muscular del movimiento de “Destrepe” de la extremidad superior

\begin{tabular}{|c|c|c|c|c|c|c|c|c|c|}
\hline & & Inic & Fin & & HOMBRO & & & CODO & \\
\hline $\begin{array}{c}\text { SUBNIVEL } \\
\text { DE } \\
\text { RESPUESTA }\end{array}$ & CÓDIGO & $\begin{array}{l}\text { Mano } \\
\text { Dcha. }\end{array}$ & $\begin{array}{l}\text { Mano } \\
\text { Izda. }\end{array}$ & Acción & $\begin{array}{c}\text { Grupos } \\
\text { musculares }\end{array}$ & $\begin{array}{c}\text { Tipo } \\
\text { Contracción }\end{array}$ & Acción & $\begin{array}{c}\text { Grupos } \\
\text { musculares }\end{array}$ & $\begin{array}{c}\text { Tipo } \\
\text { Contracción }\end{array}$ \\
\hline \multirow{15}{*}{ 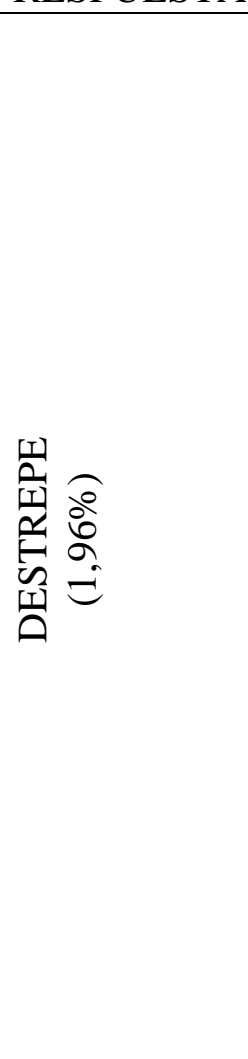 } & \multirow{2}{*}{$\begin{array}{c}\text { DC } \\
(53,7 \%)\end{array}$} & \multirow[t]{2}{*}{ 3D1D } & \multirow[t]{2}{*}{ 3I1I } & \multirow[t]{2}{*}{ Flexión } & \multirow[t]{2}{*}{ Extensores } & \multirow[t]{2}{*}{ Excéntrica } & \multirow{2}{*}{$\begin{array}{l}\text { Extensión } \\
\text { en } \\
\text { pronación }\end{array}$} & Flexores & Excéntrica \\
\hline & & & & & & & & Pronadores & Isométrica \\
\hline & \multirow{7}{*}{$\begin{array}{c}\text { DCX } \\
(14,7 \%)\end{array}$} & \multirow[t]{4}{*}{ 3D2D } & \multirow[t]{4}{*}{ 3I2I } & Flexión & Extensores & Excéntrica & \multirow[t]{3}{*}{ Extensión } & \multirow[t]{3}{*}{ Flexores } & \multirow[t]{3}{*}{ Excéntrica } \\
\hline & & & & Abducción & Aductores & Excéntrica & & & \\
\hline & & & & \multirow{2}{*}{$\begin{array}{c}\text { Rotación } \\
\text { externa }\end{array}$} & \multirow{2}{*}{$\begin{array}{l}\text { Rotadores } \\
\text { internos }\end{array}$} & \multirow[t]{2}{*}{ Excéntrica } & & & \\
\hline & & & & & & & Supinación & Pronadores & Excéntrica \\
\hline & & \multirow[t]{3}{*}{ 3D1I } & \multirow[t]{3}{*}{ 3I1D } & Flexión & Extensores & Excéntrica & \multirow{3}{*}{$\begin{array}{l}\text { Extensión } \\
\text { en } \\
\text { pronación }\end{array}$} & \multirow[t]{2}{*}{ Flexores } & \multirow[t]{2}{*}{ Excéntrica } \\
\hline & & & & Aducción & Aductores & Concéntrica & & & \\
\hline & & & & $\begin{array}{c}\text { Flexión } \\
\text { horizontal }\end{array}$ & $\begin{array}{c}\text { Flexores } \\
\text { horizontales }\end{array}$ & Concéntrica & & Pronadores & Isométrica \\
\hline & \multirow{2}{*}{$\begin{array}{c}\mathrm{DL} \\
(21,0 \%)\end{array}$} & \multirow[t]{2}{*}{$4 \mathrm{D} 2 \mathrm{D}$} & \multirow[t]{2}{*}{$4 \mathrm{I} 2 \mathrm{I}$} & \multirow[t]{2}{*}{ Abducción } & \multirow[t]{2}{*}{ Aductores } & \multirow[t]{2}{*}{ Excéntrica } & \multirow{2}{*}{$\begin{array}{l}\text { Extensión } \\
\text { en } \\
\text { pronación }\end{array}$} & Flexores & Excéntrica \\
\hline & & & & & & & & Pronadores & Concéntrica \\
\hline & \multirow{4}{*}{$\begin{array}{c}\mathrm{DLX} \\
(10,5 \%)\end{array}$} & \multirow[t]{2}{*}{ 4D1D } & \multirow[t]{2}{*}{$4 \mathrm{III}$} & \multirow{2}{*}{$\begin{array}{c}\text { Flexión } \\
\text { horizontal }\end{array}$} & \multirow{2}{*}{$\begin{array}{c}\text { Extensores } \\
\text { horizontales }\end{array}$} & \multirow[t]{2}{*}{ Excéntrica } & \multirow{2}{*}{$\begin{array}{l}\text { Extensión } \\
\text { en } \\
\text { pronación }\end{array}$} & Flexores & Excéntrica \\
\hline & & & & & & & & Pronadores & Concéntrica \\
\hline & & 4D1I & 4I1D & Flexión & Extensores & Excéntrica & Extensión & Flexores & Excéntrica \\
\hline & & & & horizontal & horizontales & & $\begin{array}{c}\text { en } \\
\text { pronación }\end{array}$ & Pronadores & Concéntrica \\
\hline
\end{tabular}


De Benito, A.M.; Sedano, S.; Redondo, J.C.; Cuadrado, G. (2013). Análisis cualitativo de las implicaciones musculares de la escalada deportiva de alto nivel en competición. RICYDE. Rev. int. cienc. deporte. 32(9), 154-180. http://dx.doi.org/10.5232/ricyde2013.03205

La tabla 17 presenta el análisis de las implicaciones musculares que subyacen a los movimientos de progresión realizados por la extremidad inferior y respecto a los grupos musculares que actúan respecto a las articulaciones de la cadera y la rodilla.

Tabla 17: Implicación muscular del movimiento de “Progresión” de la extremidad inferior

\begin{tabular}{|c|c|c|c|c|c|c|c|c|c|}
\hline & & \multicolumn{2}{|c|}{ Inicio-Fin } & \multicolumn{3}{|c|}{ CADERA } & \multicolumn{3}{|c|}{ RODILLA } \\
\hline $\begin{array}{c}\text { SUBNIVEL } \\
\text { DE } \\
\text { RESPUESTA }\end{array}$ & CÓDIGO & $\begin{array}{c}\text { Pie } \\
\text { Dcho. }\end{array}$ & $\begin{array}{l}\text { Pie } \\
\text { Izdo. }\end{array}$ & Acción & $\begin{array}{c}\text { Grupos } \\
\text { musculares }\end{array}$ & $\begin{array}{c}\text { Tipo } \\
\text { Contracción }\end{array}$ & Acción & $\begin{array}{c}\text { Grupos } \\
\text { musculares }\end{array}$ & $\begin{array}{c}\text { Tipo } \\
\text { Contracción }\end{array}$ \\
\hline \multirow{10}{*}{ 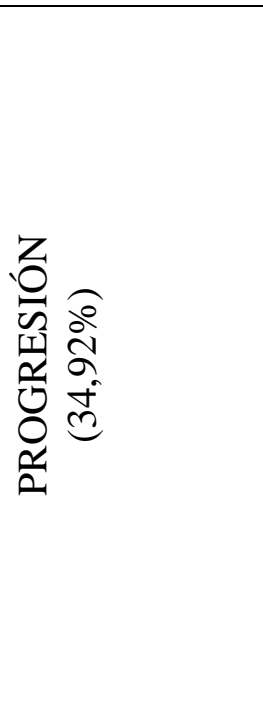 } & $\begin{array}{c}\text { PC } \\
(59,6 \%)\end{array}$ & 5D7D & 5I7I & Extensión & Extensores & Concéntrica & Extensión & Extensores & Concéntrica \\
\hline & \multirow{4}{*}{$\begin{array}{c}\text { PCX } \\
(18,6 \%)\end{array}$} & 5D5I & 5I5D & Aducción & Aductores & Concéntrica & No mov. & Extensores & Isométrica \\
\hline & & \multirow[t]{3}{*}{ 5D7I } & \multirow[t]{3}{*}{ 5I7D } & Extensión & Extensores & Concéntrica & \multirow[t]{3}{*}{ Extensión } & \multirow[t]{3}{*}{ Extensores } & \multirow[t]{3}{*}{ Concéntrica } \\
\hline & & & & Aducción & Aductores & Concéntrica & & & \\
\hline & & & & $\begin{array}{l}\text { Rotación } \\
\text { interna }\end{array}$ & $\begin{array}{c}\text { Rotadores } \\
\text { internos }\end{array}$ & Concéntrica & & & \\
\hline & \multirow{2}{*}{$\begin{array}{c}\text { PL } \\
(11,1 \%)\end{array}$} & \multirow[t]{2}{*}{ 6D8D } & \multirow[t]{2}{*}{6 6I8I } & Extensión & Extensores & Concéntrica & \multirow[t]{2}{*}{ Extensión } & \multirow[t]{2}{*}{ Extensores } & \multirow[t]{2}{*}{ Concéntrica } \\
\hline & & & & Aducción & Aductores & Concéntrica & & & \\
\hline & \multirow{3}{*}{$\begin{array}{c}\text { PLX } \\
(10,6 \%)\end{array}$} & \multirow[t]{3}{*}{ 6D7D } & \multirow[t]{3}{*}{ 6I7I } & Extensión & Extensores & Concéntrica & \multirow[t]{3}{*}{ Extensión } & \multirow[t]{3}{*}{ Extensores } & \multirow[t]{3}{*}{ Concéntrica } \\
\hline & & & & Aducción & Aductores & Concéntrica & & & \\
\hline & & & & $\begin{array}{c}\text { Rotación } \\
\text { interna }\end{array}$ & $\begin{array}{l}\text { Rotadores } \\
\text { internos }\end{array}$ & Concéntrica & & & \\
\hline
\end{tabular}


De Benito, A.M.; Sedano, S.; Redondo, J.C.; Cuadrado, G. (2013). Análisis cualitativo de las implicaciones musculares de la escalada deportiva de alto nivel en competición. RICYDE. Rev. int. cienc. deporte. 32(9), 154-180. http://dx.doi.org/10.5232/ricyde2013.03205

La tabla 18 presenta el análisis de las implicaciones musculares que subyacen a los movimientos de equilibrio realizados por la extremidad inferior y respecto a los grupos musculares que actúan respecto a las articulaciones de la cadera y la rodilla.

Tabla 18: Implicación muscular del movimiento de “Equilibrio” de la extremidad inferior

\begin{tabular}{|c|c|c|c|c|c|c|c|c|c|}
\hline & & Inic & Fin & & CADERA & & & RODILLA & \\
\hline $\begin{array}{c}\text { SUBNIVEL } \\
\text { DE } \\
\text { RESPUESTA }\end{array}$ & CÓDIGO & $\begin{array}{c}\text { Pie } \\
\text { Dcho. }\end{array}$ & $\begin{array}{l}\text { Pie } \\
\text { Izdo. }\end{array}$ & Acción & $\begin{array}{c}\text { Grupos } \\
\text { musculares }\end{array}$ & $\begin{array}{c}\text { Tipo } \\
\text { Contracción }\end{array}$ & Acción & $\begin{array}{c}\text { Grupos } \\
\text { musculares }\end{array}$ & $\begin{array}{c}\text { Tipo } \\
\text { Contracción }\end{array}$ \\
\hline \multirow{14}{*}{ 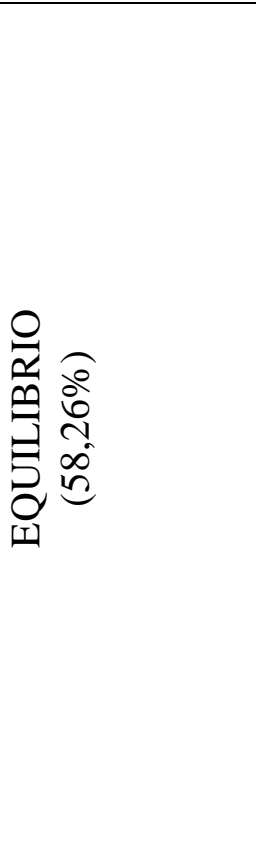 } & \multirow{2}{*}{$\begin{array}{c}\text { EQC } \\
(80,1 \%)\end{array}$} & 5D5D & 5I5I & No mov. & Extensores & Isométrica & No mov. & Extensores & Isométrica \\
\hline & & 7D7D & 7I7I & No mov. & Extensores & Isométrica & No mov. & Extensores & Isométrica \\
\hline & \multirow{4}{*}{$\begin{array}{l}\text { EQCX } \\
(4,2 \%)\end{array}$} & \multirow[t]{2}{*}{ 5I5I } & \multirow[t]{2}{*}{ 5D5D } & No mov. & Extensores & Isométrica & \multirow[t]{2}{*}{ No mov. } & \multirow[t]{2}{*}{ Extensores } & \multirow[t]{2}{*}{ Isométrica } \\
\hline & & & & No mov. & Aductores & Isométrica & & & \\
\hline & & \multirow[t]{2}{*}{ 7I7I } & \multirow[t]{2}{*}{ 7I7I } & No mov. & Extensores & Isométrica & \multirow[t]{2}{*}{ No mov. } & \multirow[t]{2}{*}{ Extensores } & \multirow[t]{2}{*}{ Isométrica } \\
\hline & & & & No mov. & Aductores & Isométrica & & & \\
\hline & \multirow{4}{*}{$\begin{array}{c}\text { EQL } \\
(15,6 \%)\end{array}$} & \multirow[t]{2}{*}{ 6D6D } & \multirow[t]{2}{*}{$6 \mathrm{I} 6 \mathrm{I}$} & No mov. & Extensores & Isométrica & \multirow[t]{2}{*}{ No mov. } & \multirow[t]{2}{*}{ Extensores } & \multirow[t]{2}{*}{ Isométrica } \\
\hline & & & & No mov. & Abductores & Isométrica & & & \\
\hline & & \multirow[t]{2}{*}{ 8D8D } & \multirow[t]{2}{*}{$8 I 8 I$} & No mov. & Extensores & Isométrica & \multirow[t]{2}{*}{ No mov. } & \multirow[t]{2}{*}{ Extensores } & \multirow[t]{2}{*}{ Isométrica } \\
\hline & & & & No mov. & Abductores & Isométrica & & & \\
\hline & \multirow{4}{*}{$\begin{array}{l}\text { EQLX } \\
(0,0 \%)\end{array}$} & \multirow[t]{2}{*}{ 6I6I } & \multirow[t]{2}{*}{ 6D6D } & No mov. & Extensores & Isométrica & \multirow[t]{2}{*}{ No mov. } & \multirow[t]{2}{*}{ Extensores } & \multirow[t]{2}{*}{ Isométrica } \\
\hline & & & & No mov. & Aductores & Isométrica & & & \\
\hline & & \multirow[t]{2}{*}{$8 \mathrm{I} 8 \mathrm{I}$} & \multirow[t]{2}{*}{ 8D8D } & No mov. & Extensores & Isométrica & \multirow[t]{2}{*}{ No mov. } & \multirow[t]{2}{*}{ Extensores } & \multirow[t]{2}{*}{ Isométrica } \\
\hline & & & & No mov. & Aductores & Isométrica & & & \\
\hline
\end{tabular}

Las tablas 19, 20 y 21 presentan el análisis de las implicaciones musculares que subyacen a los movimientos de talonamiento realizados por la extremidad inferior y respecto a los grupos musculares que actúan respecto a las articulaciones de la cadera y la rodilla. 
De Benito, A.M.; Sedano, S.; Redondo, J.C.; Cuadrado, G. (2013). Análisis cualitativo de las implicaciones musculares de la escalada deportiva de alto nivel en competición. RICYDE. Rev. int. cienc. deporte. 32(9), 154-180. http://dx.doi.org/10.5232/ricyde2013.03205

Tabla 19: Implicación muscular del movimiento de “Talonamiento” de la extremidad inferior

\begin{tabular}{|c|c|c|c|c|c|c|c|c|c|}
\hline & & Ini & Fin & & CADERA & & & RODILLA & \\
\hline $\begin{array}{c}\text { SUBNIVEL } \\
\text { DE } \\
\text { RESPUESTA }\end{array}$ & CÓDIGO & $\begin{array}{c}\text { Pie } \\
\text { Dcho. }\end{array}$ & $\begin{array}{l}\text { Pie } \\
\text { Izdo. }\end{array}$ & Acción & $\begin{array}{c}\text { Grupos } \\
\text { musculares }\end{array}$ & $\begin{array}{c}\text { Tipo } \\
\text { Contracción }\end{array}$ & Acción & $\begin{array}{c}\text { Grupos } \\
\text { musculares }\end{array}$ & $\begin{array}{c}\text { Tipo } \\
\text { Contracción }\end{array}$ \\
\hline \multirow{16}{*}{ 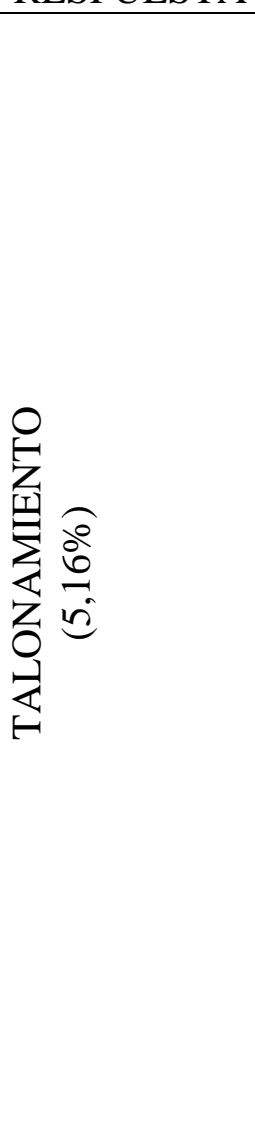 } & \multirow{3}{*}{$\begin{array}{c}\text { TC } \\
(14,8 \%)\end{array}$} & 3D5D & $3 I 5 I$ & Extensión & Extensores & Concéntrica & Flexión & Flexores & Concéntrica \\
\hline & & \multirow[t]{2}{*}{ 3D7D } & \multirow[t]{2}{*}{ 3I7I } & \multirow[t]{2}{*}{ Extensión } & \multirow[t]{2}{*}{ Extensores } & \multirow[t]{2}{*}{ Concéntrica } & Flexión & Flexores & Concéntrica \\
\hline & & & & & & & Extensión & Extensores & Concéntrica \\
\hline & \multirow{13}{*}{$\begin{array}{c}\text { TCX } \\
(8,0 \%)\end{array}$} & \multirow[t]{2}{*}{ 3D4D } & \multirow[t]{2}{*}{ 3I4I } & No mov. & Extensores & Isométrica & \multirow[t]{2}{*}{ No mov. } & \multirow[t]{2}{*}{ Flexores } & \multirow[t]{2}{*}{ Isométrica } \\
\hline & & & & Abducción & Abductores & Concéntrica & & & \\
\hline & & \multirow[t]{3}{*}{ 3D6D } & \multirow[t]{3}{*}{ 3I6I } & Extensión & Extensores & Concéntrica & \multirow[t]{3}{*}{ Extensión } & \multirow[t]{3}{*}{ Extensores } & \multirow[t]{3}{*}{ Concéntrica } \\
\hline & & & & Abducción & Abductores & Concéntrica & & & \\
\hline & & & & $\begin{array}{l}\text { Rotación } \\
\text { externa }\end{array}$ & $\begin{array}{c}\text { Rotadores } \\
\text { externos }\end{array}$ & Concéntrica & & & \\
\hline & & \multirow[t]{3}{*}{ 3D8D } & \multirow[t]{3}{*}{ 3I8I } & Extensión & Extensores & Concéntrica & \multirow[t]{3}{*}{ Extensión } & \multirow[t]{3}{*}{ Extensores } & \multirow[t]{3}{*}{ Concéntrica } \\
\hline & & & & Abducción & Abductores & Concéntrica & & & \\
\hline & & & & $\begin{array}{c}\text { Rotación } \\
\text { externa }\end{array}$ & $\begin{array}{c}\text { Rotadores } \\
\text { externos }\end{array}$ & Concéntrica & & & \\
\hline & & \multirow[t]{2}{*}{ 3D3I } & \multirow[t]{2}{*}{ 3I3D } & No mov. & Extensores & Isométrica & \multirow[t]{2}{*}{ No mov. } & \multirow[t]{2}{*}{ Flexores } & \multirow[t]{2}{*}{ Isométrica } \\
\hline & & & & $\begin{array}{c}\text { Rotación } \\
\text { externa }\end{array}$ & $\begin{array}{c}\text { Rotadores } \\
\text { externos }\end{array}$ & Concéntrica & & & \\
\hline & & \multirow[t]{3}{*}{ 3D5I } & \multirow[t]{3}{*}{ 3I5D } & Extensión & Extensores & Concéntrica & \multirow[t]{2}{*}{ Flexión } & \multirow[t]{2}{*}{ Flexores } & \multirow[t]{2}{*}{ Concéntrica } \\
\hline & & & & Aducción & Aductores & Concéntrica & & & \\
\hline & & & & $\begin{array}{c}\text { Rotación } \\
\text { externa }\end{array}$ & $\begin{array}{c}\text { Rotadores } \\
\text { externos }\end{array}$ & Concéntrica & Extensión & Extensores & Concéntrica \\
\hline
\end{tabular}


De Benito, A.M.; Sedano, S.; Redondo, J.C.; Cuadrado, G. (2013). Análisis cualitativo de las implicaciones musculares de la escalada deportiva de alto nivel en competición. RICYDE. Rev. int. cienc. deporte. 32(9), 154-180. http://dx.doi.org/10.5232/ricyde2013.03205

Tabla 20: Implicación muscular del movimiento de “Talonamiento” de la extremidad inferior (continuación de la tabla 19)

\begin{tabular}{|c|c|c|c|c|c|c|c|c|c|}
\hline & & Inic & Fin & & CADERA & & & RODILLA & \\
\hline $\begin{array}{l}\text { SUBNIVEL } \\
\text { DE } \\
\text { RESPUESTA }\end{array}$ & CÓDIGO & $\begin{array}{c}\text { Pie } \\
\text { Dcho. }\end{array}$ & $\begin{array}{l}\text { Pie } \\
\text { Izdo. }\end{array}$ & Acción & $\begin{array}{c}\text { Grupos } \\
\text { musculares }\end{array}$ & $\begin{array}{c}\text { Tipo } \\
\text { Contracción }\end{array}$ & Acción & $\begin{array}{c}\text { Grupos } \\
\text { musculares }\end{array}$ & $\begin{array}{c}\text { Tipo } \\
\text { Contracción }\end{array}$ \\
\hline \multirow{13}{*}{ 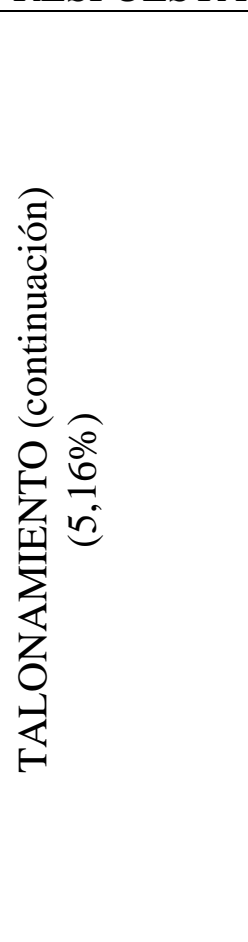 } & \multirow{8}{*}{$\begin{array}{c}\text { TCX } \\
(8,0 \%)\end{array}$} & \multirow[t]{2}{*}{ 5D6D } & \multirow[t]{2}{*}{ 5I6I } & Abducción & Abductores & Concéntrica & \multirow[t]{2}{*}{ Extensión } & \multirow[t]{2}{*}{ Extensores } & \multirow[t]{2}{*}{ Concéntrica } \\
\hline & & & & $\begin{array}{c}\text { Rotación } \\
\text { externa }\end{array}$ & $\begin{array}{c}\text { Rotadores } \\
\text { externos }\end{array}$ & Concéntrica & & & \\
\hline & & \multirow[t]{3}{*}{ 5D8D } & \multirow[t]{3}{*}{ 5I8I } & Extensión & Extensores & Concéntrica & \multirow[t]{3}{*}{ Extensión } & \multirow[t]{3}{*}{ Extensores } & \multirow[t]{3}{*}{ Concéntrica } \\
\hline & & & & Abducción & Abductores & Concéntrica & & & \\
\hline & & & & $\begin{array}{c}\text { Rotación } \\
\text { externa }\end{array}$ & $\begin{array}{c}\text { Rotadores } \\
\text { externos }\end{array}$ & Concéntrica & & & \\
\hline & & \multirow[t]{3}{*}{ 7D8D } & \multirow[t]{3}{*}{ 7I8I } & Extensión & Extensores & Concéntrica & \multirow[t]{3}{*}{ Extensión } & \multirow[t]{3}{*}{ Extensores } & \multirow[t]{3}{*}{ Concéntrica } \\
\hline & & & & Abducción & Abductores & Concéntrica & & & \\
\hline & & & & $\begin{array}{c}\text { Rotación } \\
\text { externa }\end{array}$ & $\begin{array}{c}\text { Rotadores } \\
\text { externos }\end{array}$ & Concéntrica & & & \\
\hline & \multirow{5}{*}{$\begin{array}{c}\text { TL } \\
(24,8 \%)\end{array}$} & \multirow[t]{2}{*}{ 4D6D } & \multirow[t]{2}{*}{$4 \mathrm{I} 6 \mathrm{I}$} & Extensión & Extensores & Concéntrica & \multirow[t]{2}{*}{ Extensión } & \multirow[t]{2}{*}{ Extensores } & \multirow[t]{2}{*}{ Concéntrica } \\
\hline & & & & Aducción & Aductores & Concéntrica & & & \\
\hline & & \multirow[t]{3}{*}{ 4D8D } & \multirow[t]{3}{*}{$4 \mathrm{I} 8 \mathrm{I}$} & Extensión & Extensores & Concéntrica & \multirow[t]{2}{*}{ No mov. } & \multirow[t]{2}{*}{ Flexores } & \multirow[t]{2}{*}{ Isométrica } \\
\hline & & & & Aducción & Aductores & Concéntrica & & & \\
\hline & & & & $\begin{array}{c}\text { Rotación } \\
\text { interna }\end{array}$ & $\begin{array}{l}\text { Rotadores } \\
\text { internos }\end{array}$ & Concéntrica & Extensión & Extensores & Concéntrica \\
\hline
\end{tabular}


De Benito, A.M.; Sedano, S.; Redondo, J.C.; Cuadrado, G. (2013). Análisis cualitativo de las implicaciones musculares de la escalada deportiva de alto nivel en competición. RICYDE. Rev. int. cienc. deporte. 32(9), 154-180. http://dx.doi.org/10.5232/ricyde2013.03205

Tabla 21: Implicación muscular del movimiento de “Talonamiento” de la extremidad inferior (continuación de la tablas 19 y 20)

\begin{tabular}{|c|c|c|c|c|c|c|c|c|c|}
\hline \multirow{2}{*}{$\begin{array}{c}\text { SUBNIVEL } \\
\text { DE } \\
\text { RESPUESTA }\end{array}$} & & \multicolumn{2}{|c|}{ Inicio-Fin } & \multirow{2}{*}{$\begin{array}{c}\text { CADERA } \\
\text { Acción }\end{array}$} & \multirow[b]{2}{*}{$\begin{array}{c}\text { Grupos } \\
\text { musculares }\end{array}$} & & \multirow{2}{*}{$\begin{array}{c}\text { RODILLA } \\
\text { Acción }\end{array}$} & \multirow[b]{2}{*}{$\begin{array}{c}\text { Grupos } \\
\text { musculares }\end{array}$} & \multirow[b]{2}{*}{$\begin{array}{c}\text { Tipo } \\
\text { Contracción }\end{array}$} \\
\hline & CÓDIGO & $\begin{array}{c}\text { Pie } \\
\text { Dcho. }\end{array}$ & $\begin{array}{l}\text { Pie } \\
\text { Izdo. }\end{array}$ & & & $\begin{array}{c}\text { Tipo } \\
\text { Contracción }\end{array}$ & & & \\
\hline \multirow{10}{*}{ 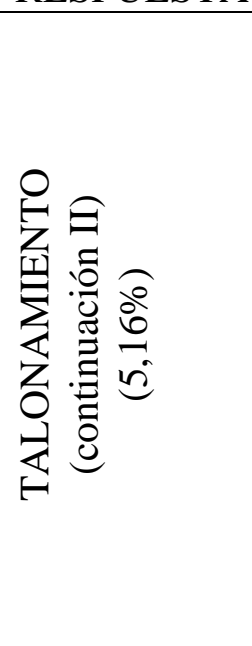 } & \multirow{10}{*}{$\begin{array}{c}\text { TLX } \\
(52,3 \%)\end{array}$} & \multirow[t]{2}{*}{ 4D3D } & \multirow[t]{2}{*}{ 4I3I } & No mov. & Extensores & Isométrica & \multirow[t]{2}{*}{ No mov. } & \multirow[t]{2}{*}{ Flexores } & \multirow[t]{2}{*}{ Isométrica } \\
\hline & & & & Aducción & Aductores & Concéntrica & & & \\
\hline & & \multirow[t]{2}{*}{ 4D5D } & \multirow[t]{2}{*}{$4 \mathrm{I} 5 \mathrm{I}$} & Extensión & Extensores & Concéntrica & \multirow[t]{2}{*}{ No mov. } & \multirow[t]{2}{*}{ Flexores } & \multirow[t]{2}{*}{ Concéntrica } \\
\hline & & & & Aducción & Aductores & Concéntrica & & & \\
\hline & & \multirow[t]{2}{*}{ 4D7D } & \multirow[t]{2}{*}{ 4I7I } & Extensión & Extensores & Concéntrica & No mov. & Flexores & Isométrica \\
\hline & & & & Aducción & Aductores & Concéntrica & Extensión & Extensores & Concéntrica \\
\hline & & \multirow[t]{2}{*}{ 6D5D } & \multirow[t]{2}{*}{$6 \mathrm{I} 5 \mathrm{I}$} & No mov. & Extensores & Isométrica & Flexión & Flexores & Concéntrica \\
\hline & & & & Aducción & Aductores & Concéntrica & $\begin{array}{c}\text { Rotación } \\
\text { externa }\end{array}$ & $\begin{array}{c}\text { Rotadores } \\
\text { externos }\end{array}$ & Concéntrica \\
\hline & & \multirow[t]{2}{*}{ 8D7D } & \multirow[t]{2}{*}{ 8I7I } & No mov. & Extensores & Isométrica & \multirow[t]{2}{*}{ No mov } & \multirow[t]{2}{*}{ Flexores } & \multirow[t]{2}{*}{ Isométrica } \\
\hline & & & & Aducción & Aductores & Concéntrica & & & \\
\hline
\end{tabular}


De Benito, A.M.; Sedano, S.; Redondo, J.C.; Cuadrado, G. (2013). Análisis cualitativo de las implicaciones musculares de la escalada deportiva de alto nivel en competición. RICYDE. Rev. int. cienc. deporte. 32(9), 154-180. http://dx.doi.org/10.5232/ricyde2013.03205

Por último, la tabla 22 presenta el análisis de las implicaciones musculares que subyacen a los movimientos de destrepe inferior realizados por la extremidad inferior y respecto a los grupos musculares que actúan respecto a las articulaciones de la cadera y la rodilla.

Tabla 22: Implicación muscular del movimiento de “Destrepe inferior” de la extremidad inferior

\begin{tabular}{|c|c|c|c|c|c|c|c|c|c|}
\hline & & Inic & -Fin & & CADERA & & & RODILLA & \\
\hline $\begin{array}{c}\text { NIVEL DE } \\
\text { RESPUESTA }\end{array}$ & CÓDIGO & $\begin{array}{c}\text { Pie } \\
\text { Dcho. }\end{array}$ & $\begin{array}{c}\text { Pie } \\
\text { Izdo. }\end{array}$ & Acción & $\begin{array}{c}\text { Grupos } \\
\text { musculares }\end{array}$ & $\begin{array}{c}\text { Tipo } \\
\text { Contracción }\end{array}$ & Acción & $\begin{array}{c}\text { Grupos } \\
\text { musculares }\end{array}$ & $\begin{array}{c}\text { Tipo } \\
\text { Contracción }\end{array}$ \\
\hline \multirow{13}{*}{ 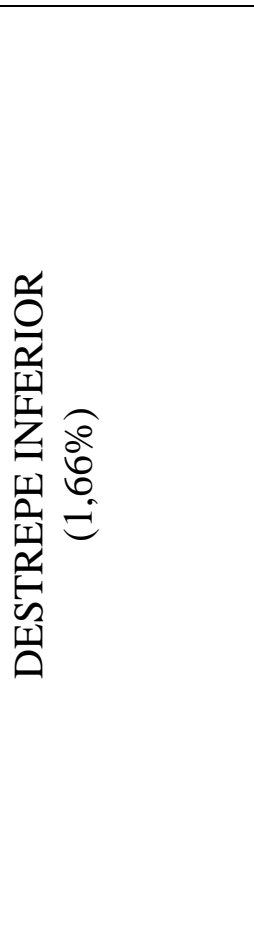 } & \multirow{2}{*}{$\begin{array}{c}\mathrm{DiC} \\
(45,8 \%)\end{array}$} & \multirow[t]{2}{*}{ 7D5D } & \multirow[t]{2}{*}{ 7I5I } & Flexión & Extensores & Excéntrica & \multirow[t]{2}{*}{ Flexión } & \multirow[t]{2}{*}{ Extensores } & \multirow[t]{2}{*}{ Excéntrica } \\
\hline & & & & Abducción & Aductores & Excéntrica & & & \\
\hline & \multirow{5}{*}{$\begin{array}{c}\mathrm{DiCX} \\
(12,5 \%)\end{array}$} & \multirow{3}{*}{$\begin{array}{l}\text { 7D6D } \\
2 \text { fases }\end{array}$} & \multirow{3}{*}{$\begin{array}{c}\text { 7I6I } \\
2 \text { fases }\end{array}$} & Flexión & Extensores & Excéntrica & Flexión & Extensores & Excéntrica \\
\hline & & & & Abducción & Aductores & Excéntrica & \multirow{2}{*}{$\begin{array}{l}\text { Rotación } \\
\text { externa }\end{array}$} & \multirow{2}{*}{$\begin{array}{c}\text { Rotadores } \\
\text { internos }\end{array}$} & \multirow[t]{2}{*}{ Excéntrica } \\
\hline & & & & $\begin{array}{l}\text { Rotación } \\
\text { externa }\end{array}$ & $\begin{array}{c}\text { Rotadores } \\
\text { internos }\end{array}$ & Excéntrica & & & \\
\hline & & \multirow[t]{2}{*}{ 7D5I } & \multirow[t]{2}{*}{ 7I5D } & \multirow[t]{2}{*}{ Flexión } & \multirow[t]{2}{*}{ Extensores } & \multirow[t]{2}{*}{ Excéntrica } & Flexión & Extensores & Excéntrica \\
\hline & & & & & & & $\begin{array}{c}\text { Rotación } \\
\text { interna }\end{array}$ & $\begin{array}{c}\text { Rotadores } \\
\text { internos }\end{array}$ & Concéntrica \\
\hline & \multirow{3}{*}{$\begin{array}{c}\text { DiL } \\
(29,2 \%)\end{array}$} & \multirow[t]{2}{*}{ 6D4D } & \multirow[t]{2}{*}{$6 \mathrm{I} 4 \mathrm{I}$} & Flexión & Extensores & Excéntrica & \multirow[t]{2}{*}{ No mov. } & \multirow[t]{2}{*}{ Flexores } & \multirow[t]{2}{*}{ Isométrica } \\
\hline & & & & $\begin{array}{c}\text { Rotación } \\
\text { externa }\end{array}$ & $\begin{array}{c}\text { Rotadores } \\
\text { internos }\end{array}$ & Excéntrica & & & \\
\hline & & 8D6D & 8I6I & Flexión & Extensores & Excéntrica & No mov. & Flexores & Isométrica \\
\hline & \multirow{3}{*}{$\begin{array}{c}\text { DiLX } \\
(12,5 \%)\end{array}$} & 8D5D & $8 I 5 I$ & Flexión & Extensores & Excéntrica & Flexión & Extensores & Excéntrica \\
\hline & & \multirow[t]{2}{*}{ 8D5I } & \multirow[t]{2}{*}{ 8I5D } & Flexión & Extensores & Excéntrica & Flexión & Extensores & Excéntrica \\
\hline & & & & Aducción & Aductores & Concéntrica & $\begin{array}{c}\text { Rotación } \\
\text { interna }\end{array}$ & $\begin{array}{c}\text { Rotadores } \\
\text { internos }\end{array}$ & Concéntrica \\
\hline
\end{tabular}

DiC: Destrepe inferior corto; DiCX: Destrepe inferior corto cruzado; DiL: Destrepe inferior largo; DiLX: Destrepe inferior largo cruzado 
De Benito, A.M.; Sedano, S.; Redondo, J.C.; Cuadrado, G. (2013). Análisis cualitativo de las implicaciones musculares de la escalada deportiva de alto nivel en competición. RICYDE. Rev. int. cienc. deporte. 32(9), 154180. http://dx.doi.org/10.5232/ricyde2013.03205

Las tablas 23 y 24 resumen las implicaciones musculares (grupo muscular y tipo de contracción) que subyacen a los diferentes movimientos de la extremidad superior en función de la acción generada.

Se crea así un entramado de relaciones cuyo conocimiento creemos necesario para la comprensión de los movimientos ejecutados en la escalada deportiva, desde un punto de vista biomecánico.

Como se puede apreciar, hay determinados grupos musculares que trabajan de las tres formas posibles (concéntrica, isométrica y excéntrica), mientras que otros sólo trabajan de una única forma.

Tabla 23: Síntesis Implicaciones musculares - movimientos de la extremidad superior

\begin{tabular}{|c|c|c|c|}
\hline \multicolumn{2}{|c|}{ HOMBRO } & \multicolumn{2}{|c|}{ CODO } \\
\hline Grupos musculares & $\begin{array}{c}\text { Tipo } \\
\text { Contracción }\end{array}$ & $\begin{array}{c}\text { Grupos } \\
\text { musculares }\end{array}$ & Tipo Contracción \\
\hline \multirow[t]{3}{*}{ Extensores } & Concéntrica & \multirow[t]{3}{*}{ Flexores } & Concéntrica \\
\hline & Isométrica & & Isométrica \\
\hline & Excéntrica & & Excéntrica \\
\hline \multirow[t]{3}{*}{ Aductores } & Concéntrica & \multirow[t]{3}{*}{ Extensores } & \multirow[t]{3}{*}{ Concéntrica } \\
\hline & Isométrica & & \\
\hline & Excéntrica & & \\
\hline \multirow[t]{3}{*}{ Abductores } & \multirow[t]{3}{*}{ Isométrica } & \multirow[t]{3}{*}{ Pronadores } & Concéntrica \\
\hline & & & Isométrica \\
\hline & & & Excéntrica \\
\hline $\begin{array}{c}\text { Flexores } \\
\text { horizontales }\end{array}$ & Concéntrica & & \\
\hline \multirow{3}{*}{$\begin{array}{c}\text { Extensores } \\
\text { horizontales }\end{array}$} & Concéntrica & & \\
\hline & Isométrica & & \\
\hline & Excéntrica & & \\
\hline $\begin{array}{l}\text { Rotadores } \\
\text { externos }\end{array}$ & Concéntrica & & \\
\hline
\end{tabular}

Tabla 24: Síntesis Implicaciones musculares - movimientos de la extremidad inferior

\begin{tabular}{|c|c|c|c|}
\hline \multicolumn{2}{|c|}{ CADERA } & \multicolumn{2}{|c|}{ RODILLA } \\
\hline Grupos musculares & $\begin{array}{c}\text { Tipo } \\
\text { Contracción } \\
\end{array}$ & $\begin{array}{c}\text { Grupos } \\
\text { musculares }\end{array}$ & Tipo Contracción \\
\hline \multirow[t]{3}{*}{ Extensores } & Concéntrica & Flexores & Isométrica \\
\hline & Isométrica & \multirow[t]{3}{*}{ Extensores } & Concéntrica \\
\hline & Excéntrica & & Isométrica \\
\hline \multirow[t]{3}{*}{ Aductores } & Concéntrica & & Excéntrica \\
\hline & Isométrica & \multirow{2}{*}{$\begin{array}{l}\text { Rotadores } \\
\text { internos }\end{array}$} & \multirow[t]{2}{*}{ Excéntrica } \\
\hline & Excéntrica & & \\
\hline \multirow[t]{2}{*}{ Abductores } & Concéntrica & & \\
\hline & Isométrica & & \\
\hline \multirow[t]{2}{*}{ Rotadores internos } & Concéntrica & & \\
\hline & Isométrica & & \\
\hline \multirow[t]{2}{*}{ Rotadores externos } & Concéntrica & & \\
\hline & Excéntrica & & \\
\hline
\end{tabular}


De Benito, A.M.; Sedano, S.; Redondo, J.C.; Cuadrado, G. (2013). Análisis cualitativo de las implicaciones musculares de la escalada deportiva de alto nivel en competición. RICYDE. Rev. int. cienc. deporte. 32(9), 154180. http://dx.doi.org/10.5232/ricyde2013.03205

\section{Discusión}

Los resultados respecto a las características de los sujetos revelan que los escaladores que componen la muestra no obedecen a un patrón antropométrico tipo, pues existe una gran dispersión de datos. En lo que respecta a la talla de los participantes, se obtuvo una altura media de 173,53 cm, cercana a los valores aportados por Watts y col. (1993), Watts y col. (1996) y Couceiro (2010), quienes obtuvieron valores de 177,8 cm., 175,6 cm. y 173,1 cm., respectivamente, para sujetos varones de similar nivel técnico. En cuanto a la envergadura, se obtuvo un valor medio de 176,99 cm., inferior a la envergadura media aportada por Couceiro (2010) de 182,2 cm.

En cuanto a la utilización de las extremidades superior e inferior en las acciones, existen porcentajes de acción muy dispares. La supremacía de los miembros superiores frente a los inferiores es una situación ampliamente contemplada en el mundo de la escalada, (Albesa y Lloveras, 1999; Núñez y col., 2005; Poblador, Gómez y Ruíz-Alejos, 2004; VicenteRodríguez, 2003). Ahora bien, dicha situación suele venir acompañada de la idea de la necesaria implicación de las extremidades inferiores en el ascenso de las vías, si queremos que éste se produzca de la forma más eficiente posible (Albesa y Lloveras, 1999; Bergua, 2009; Hoffmann, 1993; Hörst, 2007).

El análisis de los resultados de la asociación de los niveles de respuesta "posición corporal” y "extremidad" nos permite extraer lo siguiente: cuando el cuerpo adopta posiciones inclinadas (PC4) y tumbadas (PC0), la distribución de las acciones es prácticamente idéntica, encontrándonos con un 63,32\% y 63,25\% de acciones realizadas por los miembros superiores en posiciones tumbadas e inclinadas, respectivamente. Sin embargo, cuando el cuerpo se posiciona cercano a la vertical (PC9), el porcentaje de acciones efectuadas por el miembro superior desciende y recoge un valor del 57,41\%. Esta afirmación está en la línea de lo aportado por Poblador, Gómez y Ruíz-Alejos, (2004) quienes indican que cuanto mayor es la inclinación adoptada por el cuerpo a lo largo del ascenso de las vías, mayor es la participación de las extremidades superiores, en comparación con la aportación de los miembros inferiores del cuerpo y, por tanto, mayor será el desgaste asociado a la fatiga de la extremidad superior. Es por ello que: cuando la superficie del rocódromo lo permite, nos encontramos con que la posición corporal de los escaladores es prácticamente vertical, a pesar de encontrarse en un techo (inclinación $0^{\circ}$ ). De esta manera, consiguen ahorrar energía de los miembros superiores e intentan que la acción muscular requerida se reparta con cierta igualdad en ambas extremidades del cuerpo.

En cuanto a los resultados respecto a la duración de los ascensos, Fleming y Hörst (2010) aseguran que dicha categoría es una de las dimensiones críticas de la escalada deportiva. Al revisar los resultados en relación al nivel de respuesta "tiempo agrupado", se extrae que la mayor parte de los ascensos analizados poseen una duración superior a los tres minutos. Los tiempos registrados en este estudio son similares a los obtenidos por Billat, Palleja, Charlaix, Rizzardo y Janel (1995) quienes indican que las duraciones en competición de escalada pueden variar entre los 180" y los 300”. Watts (2004) en la revisión realizada, describe la duración media de una vía entre 120” y 420”. De Geus, Villanueva y Meeusen (2006) recogen duraciones de 189 " \pm 25 ” en ascenso de paredes desplomadas y de 195 " \pm 47 ” en paredes verticales. Y por último, Couceiro (2010) muestra un tiempo de ejecución medio de 160" \pm 84,34" para las vías de competición analizadas. Sin embargo, nuestros resultados son superiores a los mostrados por Watts y col. (2000) quienes recogen una duración media de 157 ” $\pm 0,41$ ” en la realización de una vía de dificultad estimada en $7 \mathrm{~b}^{+}$; y a los resultados de Sheel y col. (2003) con duraciones medias que varían entre los 90” y los 210”. Por otro lado, son inferiores a los mostrados por Egocheaga, Montoliú, González, Rodríguez, Del Valle y 
De Benito, A.M.; Sedano, S.; Redondo, J.C.; Cuadrado, G. (2013). Análisis cualitativo de las implicaciones musculares de la escalada deportiva de alto nivel en competición. RICYDE. Rev. int. cienc. deporte. 32(9), 154180. http://dx.doi.org/10.5232/ricyde2013.03205

Palenciano (2001) y Vicente-Rodríguez (2003) quienes contemplan duraciones medias de pruebas competitivas comprendidas entre 5 y 7 minutos y 318” $\pm 45,8$, respectivamente. El hecho de encontrarnos con duraciones inferiores puede deberse a dos motivos principales:

a) El primero de ellos, corresponde al hecho de encontrarnos en una modalidad competitiva en la que existe un tiempo máximo de ejecución de la vía, establecido por los jueces y contemplado por el Reglamento de competición. Dicha normativa establece que los escaladores no pueden extender su participación por encima de seis minutos para la fase de clasificación y de ocho minutos para el resto de las fases (Regla 4.8. FEDME, 2010).

b) El segundo debe su explicación a que los ascensos que integran la muestra no siempre finalizan en la máxima altura de la vía, por lo que la duración fluctúa en función de si el escalador cesó su participación por llegar al final de la misma o por haberse caído en el intento.

Los resultados obtenidos respecto a la distribución de los subniveles de respuesta están en consonancia con lo aportado por Albesa y Lloveras (1999), quienes establecen que son las acciones isométricas las más comunes y las mayores en número durante la práctica de la escalada. Según el trabajo de Dupuy y Ripoll (1989) 5/8 partes del tiempo requerido para realizar una vía se utilizan en posiciones estáticas, ya sea descansando, tomando decisiones acerca del recorrido, o protegiéndose (pasando la cuerda por los seguros intermedios). Además, otros autores argumentan que las acciones de bloqueo son la clave de este deporte (Bergua, 2009; Hoffmann, 1993; Hörst, 2007), independientemente del tipo de escalada realizada - más o menos dinámica - y de las características antropométricas que se tengan (Macià, 2002), puesto que no sólo sirven para mantener el equilibrio, sino que constituyen la posición final del movimiento de tracción (Hörst, 2007).

Ahora bien, si relacionamos los subniveles de respuesta, con la posición corporal adoptada por el escalador, obtenemos que los subniveles de respuesta "empuje”, “destrepe”, "talonamiento" y "destrepe inferior" obtienen porcentajes similares entre ellos para las tres posiciones corporales, sin embargo, los movimientos de "tracción”, “bloqueo”, "progresión” y "equilibrio" presentan porcentajes con mayor disparidad. En los casos de "tracción” y "progresión”, el mayor porcentaje se encuentra en la posición PC4, mientras que para las acciones de "bloqueo” y “equilibrio”, nos encontramos con porcentajes más elevados en PC0 y PC9, respectivamente. Los resultados aquí mostrados están en consonancia con lo citado por Vicente-Rodríguez (2003) quien obtiene mayor número de registro de acciones de "rectificación” y "reposo" en panel que en desplome. Para ello, se ha de asumir que dichas acciones podrían equivaler a los movimientos de "destrepe”, "destrepe inferior”, "bloqueo” y “equilibrio" de nuestro estudio y "panel y "desplome” a las inclinaciones corporales "PC9" y "PC4”, respectivamente.

Respecto a las subcategorías de movimiento, las acciones denominadas cortas presentan el mayor porcentaje registrado. Fleming y Hörst (2010) afirman que "los escaladores necesitan emplear las formas de movimiento biomecánicamente más ventajosas” (como por ejemplo: movimientos que conserven la fuerza del escalador). De acuerdo a este argumento, los resultados de este estudio encuentran su fundamentación en autores como Hoffmann y Kapandji (2004) quienes indican que "el máximo desarrollo de la fuerza depende del ángulo de colocación de la articulación, resultando mayor en las posiciones intermedias de la misma" (Hoffmann, 1993, 60), posición ésta que correspondería a los cuadrantes cercanos y por tanto a los movimientos denominados "cortos". Sólo el movimiento de talonamiento (T) exhibe su mayor porcentaje en su categoría "largo cruzado" (TLX). Se explica por el hecho de que su ejecución se realiza con la cadera ligeramente flexionada y la rodilla en extensión, logrando 
De Benito, A.M.; Sedano, S.; Redondo, J.C.; Cuadrado, G. (2013). Análisis cualitativo de las implicaciones musculares de la escalada deportiva de alto nivel en competición. RICYDE. Rev. int. cienc. deporte. 32(9), 154180. http://dx.doi.org/10.5232/ricyde2013.03205

así la mayor eficiencia posible de los músculos isquiotibiales, principales actores de esta acción (Kapandji, 2004, 150).

En cuanto al estudio de las implicaciones musculares que subyacen a los movimientos ejecutados por los escaladores, esperamos que las tablas aquí mostradas ayuden a analizar de forma más precisa dichos movimientos y a partir de ellas, se puedan plantear programas de entrenamiento más personalizados y eficaces.

Por último, es necesario señalar que los datos aquí mostrados respecto al número y tipo de movimientos están íntimamente relacionados con las características propias de las vías analizadas y por tanto, pueden verse modificados por las mismas. Por este motivo, se debería tener presente las características de las vías que conforman la muestra, para la comprensión de los datos aquí proporcionados.

\section{Conclusiones}

o Los grupos musculares más importantes para la escalada deportiva son: flexores del tronco, extensores, aductores y extensores horizontales del hombro, flexores y pronadores del codo, extensores, aductores, abductores, rotadores internos y rotadores externos de cadera y extensores de rodilla. Ahora bien, el tipo de contracción muscular realizada por cada uno de ellos, dependerá del movimiento requerido.

o El análisis de los ascensos en función de la extremidad ejecutante establece que la aportación de los miembros superiores es mayor que la de los miembros inferiores.

o Los movimientos más relevantes ejecutados por los escaladores de alto nivel según el orden de importancia son para la extremidad superior: bloqueo corto, tracción corta, empuje corto y destrepe corto, mientras que para la extremidad inferior son: equilibrio corto, progresión corta, talonamiento largo cruzado y destrepe inferior corto. 
De Benito, A.M.; Sedano, S.; Redondo, J.C.; Cuadrado, G. (2013). Análisis cualitativo de las implicaciones musculares de la escalada deportiva de alto nivel en competición. RICYDE. Rev. int. cienc. deporte. 32(9), 154180. http://dx.doi.org/10.5232/ricyde2013.03205

\section{Referencias}

Albesa, C. y Lloveras, P. (1999). Bases para el entrenamiento de la escalada. Madrid: Desnivel Ediciones.

Bergua, P. (2009). Entrenamiento para escalada. La técnica. Revista Digital Barrabés. <http://www.barrabes.com/revista/preparacion-fisica/2-6287/entrenamientoescalada-tecnica.htm> [Consulta: 05-12-09]

Billat, V.; Palleja, P.; Charlaix, T.; Rizzardo, P. \& Janel, N. (1995). Energy specificity of rock climbing and aerobic capacity in competitive sport rock climbers. The Journal of Sports Medicine and Physical Fitness, 35(1), 20-24.

Cohen, J.A. (1960). Coefficient of agreement for nominal scales. Educational and Psychological Measurement, 20, 37-46. Coleman, 1975.

Couceiro, J. (2010). Perfil antropométrico y respuesta psico-fisiológica en escalada deportiva en roca: diferencias entre modalidades. Tesis Doctoral para la obtención del título de Doctor en Ciencias de la Actividad Física y del Deporte, Facultad de Ciencias de la Actividad Física y del Deporte - INEF, Universidad Politécnica de Madrid, Madrid, España.

Cuadrado, G.; De Benito, A.M.; Flor, G.; Izquierdo, J.M.; Sedano, S. y Redondo, J.C. (2007). Estudio de la eficacia de dos programas de entrenamiento de la fuerza en el rendimiento de la escalada deportiva. Motricidad. European Journal of Human Movement, 19, 61-76.

De Benito, A.M. (2011). Análisis y cuantificación de las acciones técnicas de la Escalada deportiva de alto nivel y sus implicaciones musculares. Tesis Doctoral para la obtención del título de Doctor, Facultad de Educación y Trabajo Social, Universidad de Valladolid, Valladolid, España.

De Benito, A.M.; García-Tormo, J.V.; Izquierdo, J.M.; Sedano, S.; Redondo, J.C. y Cuadrado, G. (2011). Análisis de movimientos en escalada deportiva: Propuesta metodológica basada en la Metodología Observacional. Motricidad: European Journal of Human Movement, 27, 21-42.

De Geus, B.; Villanueva O'Driscoll, S. \& Meeusen, R. (2006). Influence of climbing style on physiological responses during indoor rock climbing on routes with the same difficulty. European J ournal of Applied Physiology, 98, 489-496.

Dupuy, C. \& Ripoll H. (1989) Analise des stratégies visu-motrices en escalade sportive. Revue Sciences et Motricité, 7, 19-26.

Egocheaga, J.; Montoliú, M.A.; González, V.; Rodríguez, B.; Del Valle, M. y Palenciano, L. (2001). Metabolismo energético en la escalada deportiva sobre roca y rocódromo versus escalada sobre cascada de hielo. Archivos de Medicina del Deporte, 18(81), 33-40.

España-Romero, V.; García-Artero, E.; Santaliestra-Pasias, A.M.; Gutiérrez, A.; Castillo, M.J. \& Ruiz, J.R. (2008). Hand span influences optimal grip span in boys and girls aged 6 to 12 years. Journal of Hand Surgery, 33(3), 378-384.

España-Romero, V.; Ortega, F.; García-Artero, E.; Jiménez-Pavón, D.; Gutiérrez, A.; Castillo, M.J. \& Ruiz, J.R. (2009). Climbing time to exhaustion is a determinant of climbing performance in high-level sport climbers. European Journal of Applied Physiology, 107, 517-525.

FEDME (2010). Reglamento de Competición de Escalada 2010. Madrid: FEDME. <http://www. fedme.es/?action=seccion. seccionysubseccion=143> [Consulta: $17 / 03 / 11]$ 
De Benito, A.M.; Sedano, S.; Redondo, J.C.; Cuadrado, G. (2013). Análisis cualitativo de las implicaciones musculares de la escalada deportiva de alto nivel en competición. RICYDE. Rev. int. cienc. deporte. 32(9), 154180. http://dx.doi.org/10.5232/ricyde2013.03205

Fleming, R.K. \& Hörst, E. (2010). Behaviour analysis and sports climbing. Journal of Behavioral Health and Medicine, 1(2), 143-154.

Grant, S.; Hasler, T.; Davies, C.; Aitchison, T.C.; Wilson, J. \& Whittaker, A. (2001). A comparison of the anthropometric, strength, endurance and flexibility characteristics of female elite and recreational climbers and non-climbers. Journal of Sports Science, 19(7), 499-505.

Hoffmann, M. (1993). Manual de escalada. Madrid: Desnivel Ediciones.

Hörst, E. (2007). Aprender a escalar en rocódromo. Manuales Desnivel 72. Madrid: Desnivel Ediciones.

Kapandji, A.I. (2004). Fisiología articular. (5a ed.). Madrid: Editorial Médica Panamericana, S.A.

Macià, D. (2002). Planificación del entrenamiento en escalada deportiva. Manuales Desnivel, 41. Madrid: Desnivel Ediciones.

Medina, J. y Delgado, M.A. (1999). Metodología de entrenamiento de observadores para investigadores sobre E.F. y Deporte en las que se utilice como método la observación. Motricidad. European J ournal of Human Movement, 5, 69-86.

Mermier, C.M.; Janot, J.M.; Parker, D.L. \& Swan, J.G. (2000). Physiological and anthropometric determinants of sport climbing performance. British J ournal of Sports Medicine, 34(5), 359-365.

Muro, I.; Vila, R.; Vives, J. y Gutiérrez, J.A. (1994). Estudio médico deportivo de la escalada deportiva. Apunts: Educación Física y Deportes, 31, 141-149.

Núñez, V.M.; Edir, M.; Viana, B.; Gómez, J.R.; Poblador, M. y Lancho, J.L. (2005). Estudio de la fuerza en la escalada deportiva. Archivos de Medicina del Deporte, 22(105), 27-32.

Peleteiro, J . y García-López, J. (2003). Parámetros biomecánicos en escalada deportiva y su influencia en el rendimiento. RendimientoDeportivo.com, №4.

<http://www.RendimientoDeportivo.com/N004/Artic017.htm> [Consulta: 23/01/09]

Poblador, J.A.; Gómez, E.M. y Ruíz-Alejos, C. (2004). Estudio electromiográfico de la técnica del "lanzamiento" en escalada deportiva. Lecturas: EF y Deportes (Revista Digital), № 75. <http://www.efdeportes.com/efd75/escalada.htm> [Consulta: 21/04/08]

Sheel, A.W.; Seddon, N.; Knight, A.; McKenzie, D.C. \& Warburton, D.E. (2003). Physiological response to indoor rock-climbing and their relationship to maximal cycle ergometry. Medicine y Science in Sports y Exercise, 35(7), 1225-1231.

Úbeda, A.B. (2004). Valoración de la fuerza de agarre en escaladores. Revista Internacional de Medicina y Ciencias de la Actividad Física y del Deporte, 4(14), 122 143.

Vicente-Rodríguez, G. (2003). Análisis del parámetro tiempo y cuantificación de acciones específicas en la escalada deportiva de competición. Lecturas: Educación Física y Deportes, № 59. <http://www.efdeportes.com/efd59/escalada.htm> [Consulta: 20/04/07]

Watts, P.B. (2004). Physiology of difficult rock climbing. European Journal of Applied Physiology, 91, 361-372.

Watts, P.B.; Daggett, M.; Gallagher, P. \& Wilkins, B. (2000). Metabolic response during sport rock climbing and the effects of active versus passive recovery. International J ournal of Sports Medicine, 21(3), 185-190. 
De Benito, A.M.; Sedano, S.; Redondo, J.C.; Cuadrado, G. (2013). Análisis cualitativo de las implicaciones musculares de la escalada deportiva de alto nivel en competición. RICYDE. Rev. int. cienc. deporte. 32(9), 154180. http://dx.doi.org/10.5232/ricyde2013.03205

Watts, P.B.; Jensen, R.L.; Agena, S.M.; Majchrzak, J.A.; Schellinger, R.A. \& Wubbels, C.S. (2008). Changes in EMG and finger force with repeated hangs from the hands in rock climbers. International J ournal of Exercise Science, 1(2), 62-70.

Watts, P.B.; Jensen, R.L.; Gannon, E.; Kobeinia, R.; Maynard, J. \& Sansom, J. (2008). Forearm EMG during rock climbing differs from EMG during handgrip dynamometry. International J ournal of Exercise Science, 1(1), 4-13.

Watts, P.B.; Martin, D.T. \& Durtschi, S. (1993). Anthropometric profiles of elite male and female competitive sport rock climbers. Journal of Sports Science, 11: 113-117.

Watts, P.B.; Newbury, V. \& Sulentic, J. (1996). Acute changes in handgrip strength, endurance and blood lactate with sustained sport rock climbing. Journal of Sports Medicine and Physical Fitnesss, 39: 37-41. 